\title{
MALCEV ALGEBRAS
}

\author{
BY
}

ARTHUR A. SAGLE( $\left.{ }^{1}\right)$

1. Introduction. This paper is an investigation of a class of nonassociative algebras which generalizes the class of Lie algebras. These algebras satisfy certain identities that were suggested to Malcev [7] when he used the commutator of two elements as a new multiplicative operation for an alternative algebra. As a means of establishing some notation for the present paper, a brief sketch of this development will be given here.

If $A$ is any algebra (associative or not) over a field $F$, the original product of two elements will be denoted by juxtaposition, $x y$, and the following notation will be adopted for elements $x, y, z$ of $A$ :

$$
\begin{aligned}
& \text { Commutator, } \quad x \circ y=(x, y)=x y-y x ; \\
& \text { Associator, } \quad(x, y, z)=(x y) z-x(y z) ; \\
& \text { Jacobian, } \quad J(x, y, z)=(x y) z+(y z) x+(z x) y .
\end{aligned}
$$

An alternative algebra $A$ is a nonassociative algebra such that for any elements $x_{1}, x_{2}, x_{3}$ of $A$, the associator $\left(x_{1}, x_{2}, x_{3}\right)$ "alternates"; that is,

$$
\left(x_{1}, x_{2}, x_{3}\right)=\epsilon\left(x_{i_{1}}, x_{i_{2}}, x_{i_{3}}\right)
$$

for any permutation $i_{1}, i_{2}, i_{3}$ of $1,2,3$ where $\epsilon$ is 1 in case the permutation is even, -1 in case the permutation is odd. If we introduce a new product into an alternative algebra $A$ by means of a commutator $x \circ y$, we obtain for any $x, y, z$ of $A$

$$
J(x, y, z)_{0}=(x \circ y) \circ z+(y \circ z) \circ x+(z \circ y) \circ x=6(x, y, z) .
$$

The new algebra thus obtained will be denoted by $A^{(-)}$. Using the preceding identity with the known identities of an alternative algebra [2] and the fact that the Jacobian is a skew-symmetric function in $A^{(-)}$, we see that $A^{(-)}$satisfies the identities

$$
\begin{aligned}
& x \circ x=0, \\
(x \circ y) \circ(x \circ z)= & ((x \circ y) \circ z) \circ x+((y \circ z) \circ x) \circ x \\
& +((z \circ x) \circ x) \circ y
\end{aligned}
$$

Presented to the Society, August 31, 1960; received by the editors March 14, 1961.

(1) This is essentially the author's Ph.D. dissertation written under Professor L. J. Paige while the author held a National Science Foundation Predoctoral Fellowship at the University of California, Los Angeles. The author would like to thank Professors A. A. Albert and L. J. Paige for the use of their unpublished paper on Malcev algebras. 
for all $x, y, z$ in $A^{(-)}$. An algebra $A$ whose multiplication satisfies (1.4) and (1.5) will be called a Malcev algebra or a Moufang-Lie algebra, so named by Malcev.

Since any associative algebra is an alternative algebra $A$, the Malcev algebra $A^{(-)}$is a natural generalization of a Lie algebra. Furthermore the algebra $A^{(-)}$of a Cayley-Dickson algebra yields a Malcev algebra which is not a Lie algebra. It is an interesting question, and as yet unsolved, whether or not all Malcev algebras are subalgebras of $A^{(-)}$for some alternative algebra $A$. The corresponding question for Lie algebras is of course answered in the affirmative by the Birkhoff-Witt Theorem.

Throughout this paper the algebras considered are assumed to be finite dimensional, although it should be clear when this restriction is not necessary. In $\$ 2$, many basic identities that are a consequence of (1.4) and (1.5) are derived. These identities yield the result that the linear subspace, $J(A, A, A)$, spanned by all elements of the form $J(x, y, z)$ for $x, y, z$ in $A$ is an ideal of $A$. As we shall see $J(A, A, A)$ is the most important ideal of $A$ when $A$ is not a Lie algebra. In further attempts to measure the "Lie-ness" of a Malcev algebra an extremely useful linear transformation, $\Delta(x, y)$, is given by $z \Delta(x, y)$ $=J(z, x, y)$ for all $x, y, z$ in $A$. The identities show that the linear space, $\Delta(A, A)$, spanned by all such $\Delta(x, y)$ 's is actually a Lie algebra under commutation. $\$ 3$ is concerned with examples of Malcev algebras. In particular the Cayley-Dickson algebra $A$, its associated Malcev algebra $A^{(-)}$and a seven dimensional simple Malcev algebra $A^{*}$ obtained from $A^{(-)}$are all discussed in detail. It is conjectured that $A^{*}$ is the only simple Malcev algebra which is not a simple Lie algebra. In $\$ 4$ results for Malcev algebras analogous to those for alternative algebras are discussed. Corresponding to Artin's Theorem for alternative algebras, namely, the subalgebra generated by two elements of an alternative algebra is associative, it is shown that any two elements of a Malcev algebra generate a Lie subalgebra.

$\$ 5$ is concerned with subspaces of a Malcev algebra which are invariant under the Lie algebra $\Delta(A, A)$ or invariant under the Lie transformation algebra $\mathscr{L}(A)$ [8]. It is shown that $N=\{x \in A: x \Delta(A, A)=0\}$, called the $J$ nucleus of $A$, is an ideal of $A$ and furthermore using the definition that a semi-simple Malcev algebra is a direct sum of simple algebras, it is shown that $A$ is a semi-simple Malcev algebra if and only if $\mathscr{L}(A)$ is completely reducible in $A$. In this case $A=N \oplus J(A, A, A)$ where the $J$-nucleus $N$ is a semi-simple Lie subalgebra of $A$ and $J(A, A, A)$ is a semi-simple subalgebra of $A$ which is not zero unless $A$ is a Lie algebra. A concept similar to that of solvability in a Lie algebra is defined in $\S 6$ : an ideal $B$ of a Malcev algebra $A$ is called $J$-potent if defining $J^{1}(B)=J(B, B, B)$ and $J^{k+1}(B)=J\left(J^{k}(B), J^{k}(B), J^{k}(B)\right)$ there exists an $N \geqq 2$ such that $J^{N}(B)=0$. It is shown that a maximal $J$ potent ideal, $\Im(A)$, exists and contains the maximal solvable ideal, $S$, of a Malcev algebra $A$ when $S$ is not a Lie algebra. Also if $A$ is a semi-simple, 
non-Lie, Malcev algebra, then $\Im(A)=0$. To prove the converse, an analogue to Engel's Theorem for Lie algebras seems necessary, however, further results indicate that the converse might not be true.

$\$ 7$ is concerned with the radical of a Malcev algebra $A$ which is defined to be the minimal ideal $\mathfrak{R}$ of $A$ such that $A / \mathfrak{N}$ is zero or semi-simple. To investigate the radical, more relationships between $A$ and $\mathscr{L}(A)$ are obtained and using the results of $\$ 5$ we prove the important theorem: if $A$ is a Malcev algebra of characteristic zero, then $A$ is semi-simple if and only if $\mathscr{L}(A)$ is a semi-simple Lie algebra. From this we obtain that if $A$ is a Malcev algebra of characteristic zero, then $\mathfrak{N}=A \mathcal{S}$ where $S$ is the radical of $\mathscr{L}(A)$. An interesting result relating many of the preceding concepts is the chain of inclusions $J^{1}(S) \subset J^{1}(\Im(A)) \subset A \sigma \subset A S=\Re$ where $\sigma$ is the radical of $\Delta(A, A)$ and $A \sigma$ is an ideal of $A$. Finally in this section the symmetric bilinear form $f(x, y)$ $=$ trace $\left(R_{x} R_{y}\right)$ is introduced in a Malcev algebra and it is shown that $f(x, y)$ is actually an invariant form. This result yields the analogue of Dieudonnés Theorem for Lie algebras: if $A$ is a Malcev algebra with nondegenerate trace form, then $A$ is semi-simple.

In $§ 8$, derivations are discussed and it is shown that the linear transformation $D(x, y)=\left(R_{x}, R_{y}\right)+R_{x y}$ is a derivation. Furthermore $D$ is an innerderivation [8] of a Malcev algebra $A$ if and only if $D=R_{n}+\sum_{i} D\left(x_{i}, y_{i}\right)$ where $x_{i}, y_{i}$ are arbitrary in $A$ and $n$ is arbitrary in the $J$-nucleus $N$. The derivations of the seven dimensional Malcev algebra $A^{*}$ are discussed in detail and it is shown that the derivations of $A^{*}$ form a simple Lie algebra isomorphic to the exceptional simple Lie algebra $G_{2}$. Finally in $\$ 9$, the holomorph $\mathfrak{H C}$ of a Malcev algebra is defined similarly to that of a Lie algebra: let $D$ be the derivation algebra of a Malcev algebra $A$. Form $\mathfrak{H C}=\mathscr{D} \oplus A$ and define multiplication $*$ on $\mathfrak{H C}$ by $\left(D_{1}+a_{1}\right) *\left(D_{2}+a_{2}\right)=\left(D_{1}, D_{2}\right)+a_{1} D_{2}-a_{2} D_{1}+a_{1} a_{2}$. The holomorph of a Malcev algebra is not as useful as that of a Lie algebra because of the restrictive result: $\mathcal{H C}$ is a Malcev algebra if and only if for all $x, y, z$ in $A$ and $D$ in $D, J(x, y, z) D=-J(x, y, z D)$.

2. Fundamental identities. The principal concern of this section will be the derivation of simple results that follow immediately from the definition of a Malcev algebra.

Definition. A Malcev algebra $A$ over a field $F$ is a nonassociative algebra, that is, a vector space over $F$ with a distributive multiplication defined on it satisfying

$$
\begin{gathered}
x^{2}=0, \quad x y=-y x \\
x y \cdot x z=(x y \cdot z) x+(y z \cdot x) x+(z x \cdot x) y
\end{gathered}
$$

for all $x, y, z$ of $A$.

It is not difficult to verify that any Lie algebra is a Malcev algebra; however, this result is an immediate consequence of the following 
LEMMa 2.3. The identity (2.2) of a Malcev algebra is equivalent to either of the following identities

$$
\begin{aligned}
& J(x, y, x z)=J(x, y, z) x, \\
& J(x, x y, z)=J(x, y, z) x .
\end{aligned}
$$

Proof. Assume (2.2), then $J(x, y, x z)=x y \cdot x z+(y \cdot x z) x+(x z \cdot x) y$. Now replace $x y \cdot x z$ by the right side of equation (2.2) to obtain $J(x, y, x z)=(x y \cdot z) x$ $+(y z \cdot x) x+(z x \cdot x) y+(y \cdot x z) x+(x z \cdot x) y$ or, $J(x, y, x z)=(x y \cdot z+y z \cdot x+z x \cdot y) x$ $=J(x, y, z) x$.

Thus (2.2) implies (2.4). The converse merely requires a rearrangement of terms. Similarly, it can be shown that (2.4) and (2.5) are equivalent.

If the identities (2.4) and (2.5) are combined, we obtain the identity

$$
J(x, x y, z)=J(x, y, x z)
$$

for any Malcev algebra. However, Malcev [7] has shown that (2.6) does not imply (2.2).

The linearized form of (2.4) is obtained by replacing $x$ by $x+w$ in (2.4) to obtain $J(x+w, y,(x+w) z)=J(x+w, y, z)(x+w)$ or, $J(x, y, x z)+J(x, y, w z)$ $+J(w, y, x z)+J(w, y, w z)=J(x, y, z) x+J(x, y, z) w+J(w, y, z) x+J(w, y, z) w$ by repeated application of the linearity of $J(a, b, c)$ in each argument. Two applications of (2.4) then yield

$$
J(x, y, w z)+J(w, y, x z)=J(x, y, z) w+J(w, y, z) x
$$

for all $x, y, z, w$ of $A$.

A similar linearization process for equations (2.5) and (2.6) yields

$$
\begin{aligned}
& J(x, w y, z)+J(w, x y, z)=J(x, y, z) w+J(w, y, z) x ; \\
& J(x, w y, z)+J(w, x y, z)=J(x, y, w z)+J(w, y, x z)
\end{aligned}
$$

for all $x, y, z, w$ of $A$.

The linear transformation $R_{x}$, where $x$ is an arbitrary element of a Malcev algebra $A$, is defined by the equations $a R_{x}=a x=-x a$ for all $a$ in $A$. Note that $R_{x}+R_{y}=R_{x+\nu}$ because of the distributive law of an algebra. For any $p, q, r, s$ in $A$, we have $J(p, q, r)=p\left(R_{q} R_{r}-R_{r} R_{q}-R_{q r}\right)$ and $J(p, q, r) s$ $=p\left(R_{q} R_{r} R_{\mathrm{s}}-R_{r} R_{q} R_{s}-R_{q r} R_{\mathrm{s}}\right)$.

Lemma 2.10. Let $A$ be a Malcev algebra of characteristic not 2. For elements $x, y, z, w$ of $A$, we have the following identities:

(2.14) $2 w J(x, y, z)=J(w, x, y z)+J(w, y, z x)+J(w, z, x y)$, 
$3 J(w x, y, z)=J(x, y, z) w-J(y, z, w) x-2 J(z, w, x) y+2 J(w, x, y) z$

if $A$ is of characteristic not 3,

$$
\begin{aligned}
J(J(x, y, z), x, y) & =3 x y \cdot J(x, y, z) \text { if } A \text { is of characteristic not } 3, \\
\operatorname{trace}\left(R_{z} R_{y z}\right) & =\operatorname{trace}\left(R_{z y} R_{x}\right) .
\end{aligned}
$$

Proofs. For (2.11), we begin with the identity $w R_{J(x, y, z)}=w J(x, y, z)$. Now apply (2.7) to obtain $w R_{J(x, y, z)}=J(w, y, z) x-J(w, y, x z)-J(x, y, w z)$ $=w\left(R_{y} R_{z} R_{x}-R_{y z} R_{x}-R_{z} R_{y} R_{x}-R_{y} R_{x z}+R_{y} \cdot x_{z}+R_{x z} R_{y}+R_{z} R_{x y}+R_{z} R_{y} R_{x}-R_{z} R_{x} R_{y}\right)$ $=w\left[\left(R_{y}, R_{z} R_{x}\right)+\left(R_{x z}, R_{y}\right)+R_{z} R_{x y}-R_{y z} R_{x}+R_{y} \cdot x z\right]$. Now (2.11) follows immediately.

For (2.12), note that by an appropriate change of symbols in equation (2.11) the following identities arise:

$$
\begin{aligned}
& R_{J(y, z, x)}=\left(R_{z}, R_{x} R_{y}\right)+\left(R_{x y}, R_{z}\right)+R_{x} R_{y z}-R_{z x} R_{y}+R_{z \cdot y x}, \\
& R_{J(z, x, y)}=\left(R_{x}, R_{y} R_{z}\right)+\left(R_{z y}, R_{x}\right)+R_{y} R_{z x}-R_{x y} R_{z}+R_{x \cdot z y}
\end{aligned}
$$

Adding (2.12), (2.18) and (2.19) and using the skew-symmetry of the Jacobian in a Malcev algebra, we obtain $3 R_{J(x, y, z)}=2\left[\left(R_{x}, R_{z x}\right)+\left(R_{y}, R_{z x}\right)\right.$ $\left.+\left(R_{z}, R_{x y}\right)\right]+R_{J(x, y, z)}$. Since we are assuming that the characteristic of $A$ is not 2, identity (2.12) follows.

For (2.13), add (2.11) and (2.18) to obtain $2 R_{J(x, y, z)}=\left(R_{y}, R_{z} R_{x}\right)+\left(R_{x z}, R_{y}\right)$ $+R_{z} R_{x y}-R_{y z} R_{x}+R_{y \cdot x z}+\left(R_{z}, R_{x} R_{y}\right)+\left(R_{y x}, R_{z}\right)+R_{x} R_{y z}-R_{z x} R_{y}+R_{z} \cdot y z$ $=\left(R_{x}, R_{y z}\right)+\left(R_{y}, R_{z x}\right)+\left(R_{z}, R_{x y}\right)+R_{x y \cdot z}+R_{y z \cdot x}+R_{z x \cdot y}-R_{y z \cdot x}-\left(R_{x}, R_{y} R_{z}\right)$ $+R_{z} R_{x y}-R_{z x} R_{y}$. Using (2.12) and the fact that $R_{x y \cdot z}+R_{y z} \cdot x+R_{z x \cdot y}=R_{J(x, y, z)}$, we obtain $2 R_{J(x, y, z)}=2 R_{J(x, y, z)}-R_{y z} \cdot x-\left(R_{x}, R_{y} R_{z}\right)+R_{z} R_{x y}-R_{z x} R_{y}$ and therefore $R_{y z} \cdot x=R_{z} R_{x y}-R_{z x} R_{y}+\left(R_{y} R_{z}, R_{x}\right)$ which completes the proof of (2.13).

For (2.14), use identity (2.12) to obtain $2 w J_{(x, y, z)}=w R_{J(x, y, z)}+w R_{J(x, y, z)}$ $=w\left[R_{x y \cdot z}+R_{y z \cdot x}+R_{z x \cdot y}+\left(R_{x}, R_{y z}\right)+\left(R_{y}, R_{z x}\right)+\left(R_{z}, R_{x y}\right)\right]$ $=w\left[\left(R_{x}, R_{y z}\right)-R_{x \cdot y z}+\left(R_{y}, R_{z x}\right)-R_{y \cdot z x}+\left(R_{z}, R_{x y}\right)-R_{z} \cdot x y\right]=w\left[\left(R_{x}, R_{y z}\right)-R_{x} \cdot y z\right]$ $+w\left[\left(R_{y}, R_{z x}\right)-R_{y} \cdot z x\right]+w\left[\left(R_{z}, \quad R_{x y}\right)-R_{z} \cdot x y\right]=J(w, \quad x, \quad y z)+J(w, \quad y, \quad z x)$ $+J(w, z, x y)$.

The proof of (2.15) is similar to that given for (2.14) and will be omitted.

For (2.16), let $u=J(x, y, z)$ and note that we need only show $J(u, y, y)$ $=3 x y \cdot u$. By definition, $J(u, x, y)=u x \cdot y+x y \cdot u+y u \cdot x$ and therefore it suffices to show that $u x \cdot y+y u \cdot x=2 x y \cdot u$. We begin with $2 x y \cdot u=2 x y \cdot J(x, y, z)$ $=J(x y, x, y z)+J(x y, y, z x)+J(x y, z, x y)$, by applying (2.14). Therefore $2 x y \cdot u$ $=J(x y, x, y z)+J(x y, y, z x)$ or, using (2.4) $2 x y \cdot u=J(x, y z, y) x-J(y, z x, x) y$ $=J(y, x, y z) x+J(x, y, z x) y=J(y, x, z) y \cdot x+J(x, y, z) x \cdot y=y u \cdot x+u x \cdot y$.

The identity (2.17) is the analogue for Malcev algebras of the invariant Killing form for Lie algebras; we shall make use of well-known properties of the trace function of matrices. Of course for this verification, we must assume that $A$ is a finite dimensional algebra. 
We begin with (2.11), $R_{J(x, y, z)}=\left(R_{y}, R_{z} R_{x}\right)+\left(R_{z x}, R_{y}\right)+R_{z} R_{x y}-R_{y z} R_{x}+$ $R_{y \cdot x z}$. If in (2.13) we permute arguments $x$ and $y$, we obtain $R_{y \cdot x z}=R_{x y} R_{x}$ $R_{z} R_{y x}+\left(R_{y}, R_{x} R_{z}\right)$ and substitution into the previous expression yields $R_{J(x, y, z)}$ $=\left(R_{y}, R_{z} R_{x}\right)+\left(R_{x z}, R_{y}\right)+2\left(R_{z} R_{x y}-R_{y z} R_{x}\right)+\left(R_{y}, R_{x} R_{z}\right)$. The trace of any commutator is zero so that (2.12) and this expression yield trace $\left(R_{z} R_{x y}-R_{y z} R_{x}\right)$ $=0$ or trace $R_{z} R_{x y}=$ trace $R_{y z} R_{x}$. This completes the proof of Lemma 2.10.

A simple consequence of (2.17) and (2.13) is that trace $R_{x \cdot y z}=0$ for all $x, y, z$ of $A$; this could also have been seen directly from another easily verified identity

$$
2 R_{y z \cdot x}=\left(R_{y}, R_{x z}\right)+\left(R_{z}, R_{x y}\right)+\left(R_{y} R_{z}, R_{x}\right)+\left(R_{x}, R_{z} R_{y}\right) .
$$

The nonsymmetric character of the arguments in the defining relations of a Malcev algebra can be removed by

Proposition 2.21. An algebra $A$ of characteristic not 2 is a Malcev algebra if and only if $A$ satisfies $x y=-y x$ and $x y \cdot z w=x(w y \cdot z)+w(y z \cdot x)+y(z x \cdot w)$ $+z(x w \cdot y)$ for all $x, y, z, w$ in $A$.

Proof. For the implication in one direction, merely apply (2.13) to an arbitrary element $w$ in $A$ and rearrange terms. For the converse, set $w=x$ in the identity of the proposition to obtain (2.2) in the definition of a Malcev algebra.

There remain a few more useful identities for Malcev algebra which require the ideas originated in $[2 ; 6]$ for an alternative algebra and hence we define a function $G(w, x, y, z)$ by the equation

$$
J(w x, y, z)=x J(w, y, z)+J(x, y, z) w+G(w, x, y, z)
$$

for all $w, x, y, z$ in a Malcev algebra $A$.

Proposition 2.23. In a Malcev algebra of characteristic not 2 or 3 the function $G$ as defined by (2.22) is a skew-symmetric function satisfying

$$
\begin{gathered}
3 G(w, x, y, z)=2[w J(x, y, z)-x J(y, z, w)+y J(z, w, x)-z J(w, x, y)], \\
G(w, x, y, z)=2[J(w x, y, z)+J(y z, w, x)] .
\end{gathered}
$$

Proof. That $G$ is a skew-symmetric function satisfying (2.25) is proved in [6]. In order to show (2.24), note that (2.22) yields $3 G(w, x, y, z)=3 J(w x, y, z)$ $-3 x J(w, y, z)-3 J(x, y, z) w=2 J(x, y, w) z+J(x, y, z) w+J(y, w, z) x$ $+2 J(x, w, z) y-3 J(x, y, z) w-3 x J(w, y, z)$ by applying (2.15) to the term $3 J(w x, y, z)$. Combining terms, (2.24) is an immediate consequence.

A simple consequence of (2.22) and (2.25) is

$$
J(w x, y, z)=w J(x, y, z)+J(w, y, z) x-2 J(y z, w, x) .
$$

To measure the "Lie-ness" of a Malcev algebra, the following is of considerable importance: 
Definition. For elements $x, y$ of a Malcev algebra $A$, set

$$
\Delta(x, y)=\left(R_{x}, R_{y}\right)-R_{x y} .
$$

Some elementary properties of $\Delta(x, y)$ are given in

Lemma 2.28. Let $w, x, y, z$ be any elements of a Malcev algebra $A$ of characteristic not 2 or 3 . Then

$$
\begin{aligned}
z \Delta(x, y) & =J(z, x, y), \\
-\Delta(x, y) & =\Delta(-x, y)=\Delta(y, x), \\
\Delta(x, x z) & =\Delta(x, z) R_{x}=-R_{z} \Delta(x, z), \\
2 R_{J(x, y, z)} & =\Delta(x, y z)+\Delta(y, z x)+\Delta(z, x y), \\
\Delta(x, y)^{k+1} & =(-3)^{k} \Delta(x, y) R_{x y}^{k}, \quad k=0,1,2, \cdots, \\
\left(R_{w}, \Delta(y, z)\right) & =R_{w \Delta(y, z)+2 \Delta(y z, w),}, \\
2\left(R_{w}, \Delta(y, z)\right) & =-3 \Delta(w, y z)+\Delta(y, z w)+\Delta(z, w y) .
\end{aligned}
$$

Proofs. Equations (2.29) and (2.30) are clear. Equation (2.31) follows from Lemma 2.3. Equation (2.32) follows from (2.14) and (2.29). Equation (2.33) follows from (2.16) and induction. For (2.34), let $w, x, y, z$ be in $A$, then using (2.27) we have $-x R_{w} \Delta(y, z)=w z \Delta(y, z)=J(w x, y, z)$; and, using (2.26), $-x R_{w} \Delta(y, z)=w J(x, y, z)+J(w, y, z) x-2 J(y z, w, x)=w \cdot x \Delta(y, z)-x \cdot w \Delta(y, z)$ $-2 x \Delta(y z, w)=x\left[-\Delta(y, z) R_{w}-R_{w \Delta(y, z)}-2 \Delta(y z, w)\right]$. This yields $R_{w} \Delta(y, z)$ $=\Delta(y, z) R_{w}+R_{w \Delta(y, z)}+2 \Delta(y z, w)$ and therefore (2.34). Equation (2.35) follows from (2.34) and (2.32).

As an application of these identities we prove the following useful

TheоRем 2.36. Let $A$ be a Malcev algebra of characteristic not 2 or 3 and let $\Delta(A, A)$ be the linear space spanned by all the $\Delta(x, y)$ 's, where $x, y$ are in $A$. Then $\Delta(A, A)$ is a Lie algebra under commutation.

Proof. It suffices to show that $\Delta(A, A)$ is closed under commutation. First note that for any $v, w, y, z$ in $A, 2\left(R_{w}, \Delta(y, z)\right)=-3 \Delta(w, y z)+\Delta(y, z w)$ $+\Delta(z, w y)$ is in $\Delta(A, A)$, using (2.35). Secondly note that

$$
\begin{aligned}
& 4\left(R_{v},\left(R_{w}, \Delta(y, z)\right)\right. \\
& \quad=-6\left(R_{v}, \Delta(w, y z)\right)+2\left(R_{v}, \Delta(y, z w)\right)+2\left(R_{v}, \Delta(z, w y)\right)
\end{aligned}
$$

is again in $\Delta(A, A)$ by using the preceding sentence. Now (2.37) implies that $\left(\left(R_{v}, R_{w}\right), \Delta(y, z)\right)=\left(R_{v},\left(R_{w}, \Delta(y, z)\right)\right)-\left(R_{w},\left(R_{v}, \Delta(y, z)\right)\right)$ is in $\Delta(A, A)$.

Now for any $\Delta(y, w), \Delta(y, z)$ in $\Delta(A, A)$ we have $4(\Delta(v, w), \Delta(y, z))$ $=4\left(\left(R_{v}, R_{w}\right)-R_{v w}, \Delta(y, z)\right)=-4\left(R_{v w}, \Delta(y, z)\right)+4\left(\left(R_{v}, R_{w}\right), \Delta(y, z)\right)$ is in $\Delta(A, A)$, by the first part of the proof. Using the bilinearity of the commutator, we have for any $S, T$ in $\Delta(A, A)$ that $(S, T)$ is in $\Delta(A, A)$. We shall note that 


$$
\begin{aligned}
(\Delta(x, y), \Delta(u, v))= & \Delta(x \Delta(u, v), y)+\Delta(x, y \Delta(u, v))+3 R_{J(x, y, u v)} \\
& -6 \Delta(x y, u v) .
\end{aligned}
$$

This is easily obtained from (8.13) and Proposition 8.14.

3. Examples and ideals of Malcev algebras. The notation and definitions given in [1] for any nonassociative algebra $A$ will be adopted for our investigations of Malcev algebras. These are: (1) If $h\left(x_{1}, \cdots, x_{n}\right)$ is a function of $n$ indeterminates such that for any $n$ subsets $B_{i}$ of $A$ the elements $h\left(b_{1}, \cdots, b_{n}\right)$, for $b_{i} \in B_{i}$, are in $A$, then $h\left(B_{1}, \cdots, B_{n}\right)$ denotes the linear subspace of $A$ spanned by all of the elements $h\left(b_{1}, \cdots, b_{n}\right)$, for $b_{i} \in B_{i}$. (2) If $B$ is a subset of $A$ and $B$. is a set of linear transformations defined on $A$, then $B B$ denotes the linear subspace of $A$ spanned by all the elements $b \beta$ for $b \in B$ and $\beta \in B$. (3) A linear subspace $B$ of $A$ is called an ideal of $A$ if $B A \subset B$ and $A B \subset B$. An algebra $A$ is called simple if $A^{2} \neq 0$ and $A$ contains no proper ideals. Also an algebra $A$ is semi-simple if $A$ is the direct sum of ideals which are themselves simple algebras. For an algebra $A$, set $A^{(2)}=A \cdot A$ and $A^{(k+1)}=A^{(k)}$ $\cdot A^{(k)}$. The chain $A \supset A^{(2)} \supset \cdots \supset A^{(k)} \supset \cdots$ is called the derived series of $A$ and if there exists an integer $N$ such that $A^{(N)}=0, A$ is called solvable. An element $a$ of $A$ is an absolute divisor of zero if $a A=A a=0$. The notation $\bar{x}$ or $[x]^{-}$is used to denote elements of a quotient space $\bar{A}$ of $A$, and $\bar{B}$ or $[B]^{-}$ is used to denote subsets of $\bar{A}$.

ExAmple 3.1. The following is a solvable, non-Lie, Malcev algebra. Let $A$ have basis $\left\{e_{1}, e_{2}, e_{3}, e_{4}\right\}$ with multiplication table

\begin{tabular}{l|rrrr} 
& $e_{1}$ & $e_{2}$ & $e_{3}$ & $e_{4}$ \\
\hline$e_{1}$ & 0 & $-e_{2}$ & $-e_{3}$ & $e_{4}$ \\
$e_{2}$ & $e_{2}$ & 0 & $2 e_{4}$ & 0 \\
$e_{3}$ & $e_{3}$ & $-2 e_{4}$ & 0 & 0 \\
$e_{4}$ & $-e_{4}$ & 0 & 0 & 0
\end{tabular}

With a few calculations, one can easily show that $A$ is a Malcev algebra. However, $J\left(e_{1}, e_{2}, e_{1} e_{3}\right)=6 e_{4}=J\left(e_{1}, e_{2}, e_{3}\right) e_{1}$ and hence $A$ is not a Lie algebra. The derived series of $A$ is $A \supset\left\{e_{2}, e_{3}, e_{4}\right\} \supset\left\{e_{4}\right\} \supset 0$, and $A$ is a solvable algebra.

Example 3.2. The $A^{(-)}$algebra of a Cayley-Dickson algebra $A$. Every Cayley-Dickson algebra can be extended to an algebra containing zero divisors by a suitable extension of the base field. We shall assume this extension has been made and use the Zorn "vector-matrix" form of the Cayley-Dickson algebra possessing idempotents not the identity.

Briefly, we let the elements of the algebra $A$ be of the form

$$
\left(\begin{array}{ll}
a & \alpha \\
\beta & b
\end{array}\right)
$$


where $a, b$ are elements of a field $F$ and $\alpha, \beta$ are 3-dimensional vectors $\left(a_{1}, a_{2}, a_{3}\right)$ over $F$.

For addition, we define

$$
\left(\begin{array}{ll}
a & \alpha \\
\beta & b
\end{array}\right)+\left(\begin{array}{ll}
c & \gamma \\
\delta & d
\end{array}\right)=\left(\begin{array}{ll}
a+c & \alpha+\gamma \\
\beta+\delta & b+d
\end{array}\right)
$$

We use the notation - and $X$ for the scalar and vector product of 3dimensional vectors and define multiplication by

$$
\left(\begin{array}{ll}
a & \alpha \\
\beta & b
\end{array}\right) \cdot\left(\begin{array}{ll}
c & \gamma \\
\delta & d
\end{array}\right)=\left(\begin{array}{cc}
a c+\alpha \cdot \delta & a \gamma+d \alpha-\beta \times \delta \\
c+b \delta+\alpha \times \gamma & \beta \cdot \gamma+b d
\end{array}\right)
$$

$A$ is an alternative algebra and we define a new multiplication $\circ$ by $A \circ B=A B-B A$, or

$$
\begin{aligned}
& \left(\begin{array}{ll}
a & \alpha \\
\beta & b
\end{array}\right) \circ\left(\begin{array}{ll}
c & \gamma \\
\delta & d
\end{array}\right) \\
& \quad=\left(\begin{array}{cc}
\alpha \cdot \delta-\beta \cdot \gamma & (a-b) \gamma+(d-c) \alpha-2 \beta \times \delta \\
(c-d) \beta+(b-a) \delta+2 \alpha \times \gamma & -(\alpha \cdot \delta-\beta \cdot \gamma)
\end{array}\right) .
\end{aligned}
$$

The algebra $A^{(-)}$, with multiplication defined by (3.3), is a Malcev algebra and we choose as a basis for $A^{(-)}$the elements

$$
\begin{array}{lll}
e_{0}=\left(\begin{array}{ll}
1 & 0 \\
0 & 1
\end{array}\right), & e_{1}=\left(\begin{array}{cc}
1 & 0 \\
0 & -1
\end{array}\right), & e_{2}=\left(\begin{array}{ll}
0 & (1,0,0) \\
0 & 0
\end{array}\right), \\
e_{3}=\left(\begin{array}{ll}
0 & (0,1,0) \\
0 & 0
\end{array}\right), & e_{4}=\left(\begin{array}{cc}
0 & (0,0,1) \\
0 & 0
\end{array}\right), & e_{5}=\left(\begin{array}{cc}
0 & 0 \\
(1,0,0) & 0
\end{array}\right), \\
e_{6}=\left(\begin{array}{ll}
0 & 0 \\
(0,1,0) & 0
\end{array}\right), & e_{7}=\left(\begin{array}{ccc}
0 & 0 \\
(0,0,1) & 0
\end{array}\right) .
\end{array}
$$

For all $x$ in $A^{(-)}, e_{0} \circ x=0$ and hence we shall consider the algebra $A^{*}=A^{(-)} /\left\{e_{0}\right\}$. We identify $e_{i}$ in $A^{(-)}$with the coset $e_{i}+\left\{e_{0}\right\}$ in $A^{*}$ and denoting multiplication by juxtaposition we have the following multiplication table for fields of characteristic not 2 :

\begin{tabular}{r|rrrrrrr} 
& $e_{1}$ & $e_{2}$ & $e_{3}$ & $e_{4}$ & $e_{5}$ & $e_{6}$ & $e_{7}$ \\
\hline$e_{1}$ & 0 & $2 e_{2}$ & $2 e_{3}$ & $2 e_{4}$ & $-2 e_{5}$ & $-2 e_{6}$ & $-2 e_{7}$ \\
$e_{2}$ & $-2 e_{2}$ & 0 & $2 e_{7}$ & $-2 e_{6}$ & $e_{1}$ & 0 & 0 \\
$e_{3}$ & $-2 e_{3}$ & $-2 e_{7}$ & 0 & $2 e_{5}$ & 0 & $e_{1}$ & 0 \\
$e_{4}$ & $-2 e_{4}$ & $2 e_{6}$ & $-2 e_{5}$ & 0 & 0 & 0 & $e_{1}$ \\
$e_{5}$ & $2 e_{5}$ & $-e_{1}$ & 0 & 0 & 0 & $-2 e_{4}$ & $2 e_{3}$ \\
$e_{6}$ & $2 e_{6}$ & 0 & $-e_{1}$ & 0 & $2 e_{4}$ & 0 & $-2 e_{2}$ \\
$e_{7}$ & $2 e_{7}$ & 0 & 0 & $-e_{1}$ & $-2 e_{3}$ & $2 e_{2}$ & 0
\end{tabular}


It is an easy matter to prove $A^{*}$ is a simple algebra. If $B$ is an ideal containing an element $t=\sum_{i=1}^{7} t_{i} e_{i}$, where $t_{1} \neq 0$, then $4 t-\left(t e_{1}\right) e_{1}=4 t_{1} e_{1}$ is in $B$ and therefore $e_{1}$ is in $B$. Consequently, from the above multiplication table, $B=A^{*}$. An ideal $B$ of $A^{*}$ always contains such an element because if $s=\sum_{i=2}^{7} s_{i} e_{i}$, (where $s_{2} \neq 0$ for example), then $e_{5} s=-s_{2} e_{1}$ tother terms.

EXAMPLE 3.4. In the theory of Lie algebras, the product of two ideals is again an ideal. However, the following is an example of a Malcev algebra $A$ with ideals $B$ and $C$ which are Lie algebras, yet $B C$ is not an ideal of $A$. Let $A$ have basis $\left\{e_{1}, e_{2}, e_{3}, e_{4}, e_{5}\right\}$ with multiplication table

\begin{tabular}{l|rrrrr} 
& $e_{1}$ & $e_{2}$ & $e_{3}$ & $e_{4}$ & $e_{5}$ \\
\hline$e_{1}$ & 0 & 0 & 0 & $e_{2}$ & 0 \\
$e_{2}$ & 0 & 0 & 0 & 0 & $e_{3}$ \\
$e_{3}$ & 0 & 0 & 0 & 0 & 0 \\
$e_{4}$ & $-e_{2}$ & 0 & 0 & 0 & 0 \\
$e_{5}$ & 0 & $-e_{3}$ & 0 & 0 & 0
\end{tabular}

Then $B=\left\{e_{1}, e_{2}, e_{3}\right\}$ and $C=\left\{e_{2}, e_{3}, e_{4}\right\}$ are ideals of $A$, yet $B C=\left\{e_{2}\right\}$ is not an ideal of $A$.

We are able to provide a multiplication of ideals of a Malcev algebra with the following

TheOREM 3.5. If $B$ and $C$ are ideals of a Malcev algebra $A$ of characteristic not 2 or 3 , then $J(B, C, A)$ is an ideal of $A$.

Proof. By (2.14) we have $A J(B, C, A) \subset J(A, B, C A)+J(A, C, A B)$ $+J(A, A, B C)$. Since $B$ and $C$ are ideals of $A$, this yields $A J(B, C, A)$ $C J(A, B, C)+J(A, C, B)+J(A, A, B C)$. Thus to show $J(B, C, A)$ is an ideal of $A$, it suffices to show $J(A, A, B C) \subset J(B, C, A)$. So let $x, y$, be in $A$, $b$ in $B$ and $c$ in $C$. Then by $(2.26), J(b c, x, y)=b J(c, x, y)-c J(b, x, y)$ $-2 J(x y, b, c)$ and therefore using (2.14) we obtain that $2 J(b c, x, y)=2 b J(c, x, y)$ $-2 c J(b, x, y)-4 J(x y, b, c)=J(b, c, x y)+J(b, x, y c)+J(b, y, c x)-J(c, b, x y)$ $-J(c, x, y b)-J(c, y, b x)-4 J(x y, b, c)$ is in $J(B, C, A)$.

COROLlary 3.6. If $A$ is a Malcev algebra of characteristic not 2 or 3, then $J(A, A, A)$ is the minimal ideal of $A$ such that $A / J(A, A, A)$ is a Lie algebra.

4. Lie subalgebras of a Malcev algebra. Bruck and Kleinfeld considered associative subrings of an alternative ring in [2] and the proofs in this section are modifications of those in [2]. We shall permit the Malcev algebras in this section to have arbitrary characteristic because the only previously stated identities used (except in Theorem 4.1) are (1.3), (2.1), (2.2) and (2.22) none of which depend on the characteristic.

Definition. A subset $B$ of a Malcev algebra $A$ is a Lie subset of $A$ if 
$J(B, B, B)=0 . B$ is a maximal Lie subset of $A$ provided $B$ is a maximal subset of $A$ such that $J(B, B, B)=0$.

It is easily shown that $B$ is a maximal Lie subset of $A$ provided $J(x, B, B)$ $=0$ if and only if $x$ is in $B$. Also by using Zorn's Lemma, one can easily verify that every Lie subset is contained in a maximal Lie subset.

TheOREM 4.1. Let $A$ be a Malcev algebra such that $3 G(w, x, y, z)=0$ implies $G(w, x, y, z)=0$ for every $w, x, y, z$ in $A$. Then every maximal Lie subset $B$ of $A$ is a subalgebra of $A$.

Proof. Let $w, x, y, z$ be in $B$, then (2.24) and the hypothesis on $A$ yield $G(w, x, y, z)=0$. Now by (2.22) we have $J(w x, y, z)=0$ and since $B$ is a maximal Lie subset of $A, w x$ is in $B$. By the linearity of the Jacobian, $B$ is also a subspace of $A$.

Theorem 4.2. Let $X, B, C$ be subsets of a Malcev algebra $A$ such that

$$
J(X, X, A)=J(B, B, A)=J(C, C, A)=J(X, B, C)=0 .
$$

Then the subset $D=X \cup B \cup C$ is contained in a Lie subalgebra of $A$.

Corollary 4.4. Any two elements $a, b$ of $A$ (or any three elements $a, b, c$ of $A$ satisfying $J(a, b, c)=0$ ) are contained in a Lie subalgebra of $A$.

Proof. From (4.3) and (2.22), which states that $J(w x, y, z)=x J(w, y, z)$ $+J(x, y, z) w+G(w, x, y, z)$, we have

$$
G(A, A, X, X)=G(A, A, B, B)=G(A, A, C, C)=0 .
$$

Let $K=\{k \in A: J(D, D, k)=G(X, B, C, k)=0\}$; that is,

$$
J(D, D, K)=G(X, B, C, K)=0 \text { defines } K \text {. }
$$

We shall show that $D K \subset K$. If $d$ is in $D=X \cup B \cup C$, clearly $J(D, D, d)=0$ by the hypotheses; and by $(4.5), G(X, B, C, d)=0$. Thus $d$ is in $K$; that is $D \subset K$. Furthermore, from (4.5) and (4.6), we have

$$
G(D, D, D, K)=0 \text {. }
$$

From (2.22), (4.6) and (4.7) we have

$$
J(D D, D, K)=0
$$

and for $z$ in $D \cup D D$, we have

$$
J(X K, X, z)=J(K, X, z) X+K J(X, X, z)+G(X, K, X, z)=0
$$

by using (2.22), (4.3), (4.5), (4.6) and (4.7). Thus we have

$$
J(X K, X, D)=J(X K, X, D D)=0 .
$$

Hence by $(2.22), J(B C, X, X K)=J(C, X, X K) B+C J(B, X, X K)$ 
$+G(B, C, X, X K)$, and using (4.10), we have $J(B C, X, X K)=G(B, C, X, X K)$ $=0$. Arguing similarly on $B$ and $C$ we obtain

$$
G(X, B, C, D K)=0 .
$$

Since $D \subset K,(2.22)$ yields

$$
J(D K, D, D)=J(K, D, D) D+K J(D, D, D)+G(D, K, D, D)=0,
$$

and hence by (4.6), (4.11) and (4.12) we complete the proof that $D K \subset K$.

Let

$$
M=\{m \in K: J(D, m, K)=0 \text { and } m K \subset K\} ;
$$

that is, $J(D, M, K)=0$ and $M K \subset K$, where $M \subset K$. Let

$$
S=\{s \in M: J(s, M, K)=0\}
$$

that is, $J(S, M, K)=0$, where $S \subset M$.

Note that by (4.6), $D \subset M$ and $D \subset S$. Clearly $S$ is a subspace of $A$ and we shall show that $S$ is a Lie algebra. Now since $S \subset M \subset K$ we have $J(S, S, S)=0$. So we need only show $S$ is closed under multiplication. Also since $S \subset M \subset K$ and $M K \subset K$ we have, using (2.22), (4.13) and (4.14), that $S S \subset K$ and $G(M, K, S, S)=0$. Furthermore,

$$
J(S S, M, K)=J(S, M, K) S+S J(S, M, K)+G(S, S, M, K)=0 .
$$

From $0=J(S, S, K)$ we have $S \cdot S K+S K \cdot S+K \cdot S S=0$, and since $S K \subset K$, we obtain $S S \cdot K \subset K$. Now $J(S S, M, K)=0$ implies, since $D \subset M$, that $J(S S, D, K)=0$ and therefore by (4.13), $S S \subset M$. Hence by (4.13) and (4.14), $S S C S$. This completes the proof. The corollary is an immediate consequence.

By Zorn's Lemma, every Lie subalgebra of a Malcev algebra is contained in a maximal Lie subalgebra which, we shall now show, is a maximal Lie subset of the Malcev algebra.

ThEOREM 4.16. Let $B$ be a Lie subalgebra of a Malcev algebra $A$ and let $C$ be a subset of $A$ such that $J(B, B, C)=J(C, C, A)=0$. Then $D=B \cup C$ is contained in a Lie subalgebra of $A$.

COROLlary 4.17. Every maximal Lie subalgebra of $A$ is a maximal Lie subset of $A$.

Proof. Let $K=\{k \in A: J(D, D, k)=0\}$; that is $J(D, D, K)=0$ defines $K \subset A$. We shall show $D K \subset K$. Since $J(C, C, A)=0$, we have by (2.22)

$$
\begin{aligned}
0 & =J(A A, C, C)=J(A, C, C) A+A J(A, C, C)+G(A, A, C, C) \\
& =G(A, A, C, C) .
\end{aligned}
$$

Since $B B C B C D$, we have $0=J(B B, B, K)=G(B, B, B, K)$. Therefore $0=J(B B, C, K)=J(B, C, K) B+B J(B, C, K)+G(B, B, C, K)=G(B, B, C, K)$. Thus 


$$
0=J(B B, D, K)=G(B, B, D, K) .
$$

Using (2.22), (4.18) and (4.19) we obtain

$$
G(D, D, D, K)=0 .
$$

Moreover by the hypotheses, $D \subset K$ and hence $J(D K, D, D)=K J(D, D, D)$ $+J(K, D, D) D+G(D, K, D, D)=0$. Thus by the definition of $K, D K \subset K$. Hence $D \subset M=\{m \in K: J(D, m, K)=0$ and $m K \subset K\}$. If $S=\{s \in M: J(s, M, K)=0\}$ we see as in Theorem 4.2 that $D \subset S$ and $S$ is a Lie subalgebra. For the corollary, let $B$ be a maximal Lie subalgebra of $A$ and let $J(c, B, B)=0$. To show that $c$ is in $B$, let $C=c$ in Theorem 4.16. Then $B \subset B \cup c \subset S$, where $S$ is a Lie subalgebra of $A$. Hence by the maximality of $B, B=B \cup c$ and $c$ is in $B$.

5. Invariant subspaces of the Lie algebras $\Delta(A, A)$ and $\mathscr{L}(A)$. In this section we shall consider the Lie transformation algebra, $\mathscr{L}(A)$, the Lie algebra $\Delta(A, A)$ and their invariant subspaces. Schafer [8] has defined the Lie transformation algebra of an arbitrary nonassociative algebra $A$ as follows: Let $R(A)(L(A))$ be the linear space spanned by all right (left) multiplications $R_{x}\left(L_{x}\right)$ for $x$ in $A$ and set $M=R(A)+L(A)$. Then the Lie transformation algebra, $\mathcal{L}(A)$, of $A$ is the intersection of all Lie algebras containing $M$. Defining $M_{1}=M, M_{i}=\left(M_{i-1}, M_{1}\right), i=2,3, \cdots$, Schafer has shown that $\left(M_{i}, M_{j}\right) \subset M_{i+j}$ for $i, j=1,2, \cdots$ and that $\mathscr{L}(A)=M_{1}$ $+M_{2}+\cdots$.

For the remainder of this paper we shall restrict all algebras to have characteristic not 2 or 3.

Theorem 5.1. Let $A$ be a Malcev algebra, then $\mathcal{L}(A)=R+(R, R)$ where $R=R(A)$.

Proof. Since $A$ is anti-commutative, $M_{1}=R \equiv R_{1}$ and $M_{i} \equiv R_{i}=\left(R_{i-1}, R_{i}\right)$. By (2.20), $2 R_{x y \cdot z}=\left(R_{x}, R_{y z}\right)+\left(R_{y}, R_{z x}\right)+\left(\left(R_{x}, R_{y}\right), R_{z}\right)$. Using the above notation, $R_{3} \subset R_{1}+R_{2}$. Moreover $\left(R_{2}, R_{2}\right) \subset R_{4}=\left(R_{3}, R_{1}\right) \subset\left(R_{1}+R_{2}, R_{1}\right) \subset R_{2}+R_{3}$ $C R_{1}+R_{2}+R_{1}$. Hence $R_{1}+R_{2}$ is a Lie algebra and therefore by definition $\mathcal{L}(A)=R+(R, R)$.

As an application of the Lie transformation algebra, we have the following

Theorem 5.2. Let $B$ be an ideal of a Malcev algebra $A$. The subspace $R(B)+(R(B), R(A))$ generated by all elements of the form $R_{b}+\left(R_{b^{\prime}}, R_{a}\right)$ for $b, b^{\prime}$ in $B, a$ in $A$ is an ideal of $\mathfrak{L}(A)$.

Corollary 5.3. If $A=A \cdot A$ contains no absolute divisors of zero and $\mathfrak{L}(A)$ is simple, then $A$ is simple.

Proof. From (2.20) we have

$$
\left(\left(R_{x}, R_{y}\right), R_{z}\right)=2 R_{x y \cdot z}-\left(R_{x}, R_{y z}\right)-\left(R_{y}, R_{z x}\right) .
$$


If $x \in B$ and $y, z \in A$, then $x y \cdot z \in B, y z \in A, z x \in B$. This and (5.4) imply

$$
(R(B),(R(A), R(A))) \subset R(B)+(R(B), R(A)) \text {. }
$$

If $x, z \in A$ and $y \in B$, then $x y \cdot z \in B, y z \in B, z x \in A$. This and (5.4) imply

$$
((R(A), R(B)), R(A)) \subset R(B)+(R(B), R(A)) \text {. }
$$

From the Jacobi identity for Lie algebras we see that

$$
\begin{aligned}
& ((R(B), R(A)),(R(A), R(A))) \\
& \quad=-([R(A),(R(A), R(A))], R(B))-([(R(A), R(A)), R(B)], R(A)) .
\end{aligned}
$$

Clearly $(R(A),(R(A), R(A))) \subset R(A)+(R(A), R(A))$, from Theorem 5.1, and hence using (5.5)

$$
\begin{aligned}
([R(A),(R(A), R(A))], R(B)) \\
\subset(R(A)+(R(A), R(A)), R(B)) \subset R(B)+(R(B), R(A)) .
\end{aligned}
$$

We also have $([(R(A), R(A)), R(B)], R(A)) \subset R(B)+(R(B), R(A))$ from (5.5) and (5.6), so that $([R(B)+(R(B), R(A))],[R(A)+(R(A), R(A))])$ $C(R(B), \quad R(A))+(R(B), \quad(R(A), \quad R(A)))+((R(B), R(A)),(R(A), R(A)))$ $C R(B)+(R(B), R(A))$. Hence $R(B)+(R(B), R(A))$ is an ideal of $\mathscr{L}(A)$.

For the corollary note that $R(B)+(R(B), R(A))$ is a nonzero ideal of $\mathscr{L}(A)=R(A)+(R(A), R(A))$ if $B$ is a nonzero ideal of $A$. Thus if $\mathcal{L}(A)$ is simple, $R(B)+(R(B), R(A))=R(A)+(R(A), R(A))$ and therefore $R(A)$ $C R(B)+(R(B), R(A))$. Hence $A=A \cdot A=A R(A) \subset A R(B)+A(R(B), R(A))$ $C B$.

Proposition 5.8. $\Delta(A, A)$ is an ideal in the Lie transformation algebra and $\mathscr{L}(A)=R(A)+\Delta(A, A)$.

Proof. It suffices to show $\left(R_{x}, \Delta(u, v)\right)$ and $\left(\left(R_{y}, R_{z}\right), \Delta(u, v)\right)$ are in $\Delta(A, A)$. But this follows from (2.35) and (2.38). The second part follows from Theorem 5.1 and (2.27).

Theorem 5.9. If $A$ is a Malcev algebra such that $A=J(A, A, A)$, then $\Delta(A, A)=\mathfrak{L}(A)$.

Proof. We know $\Delta(A, A) \subset \mathscr{L}(A)$. So let $R_{x}+\left(R_{y}, R_{z}\right)$ be in $\mathscr{L}(A)$ where $x, y, z$ are in $A=J(A, A, A)$. Then by (2.32), $R_{x}, R_{y}$ and $R_{z}$ are in $\Delta(A, A)$ and since $\Delta(A, A)$ is a Lie algebra under commutation, $R_{x}+\left(R_{y}, R_{z}\right)$ is in $\Delta(A, A)$.

We shall now consider $\mathscr{L}(A)$ - and $\Delta(A, A)$-invariant subspaces of a Malcev algebra. See [5] for basic properties of invariant subspaces.

Proposition 5.10. Let $A$ be an arbitrary nonassociative algebra and let $B$ be a subspace of $A$. Then $B$ is $\mathcal{L}(A)$-invariant if and only if $B$ is an ideal of $A$.

Proof. Suppose $B$ is an ideal of $A$, then $B R(A) \subset B$ and $B L(A) \subset B$. Thus 
$B M_{i} \subset B$ for all $i$ and therefore $B$ is $\mathscr{L}(A)$-invariant. Conversely, suppose $B$ is $\mathscr{L}(A)$-invariant. Then $B \mathscr{L}(A) \subset B$ and in particular, $B R(A) \subset B$ and $B L(A)$ $C B$. Therefore $B$ is an ideal of $A$.

Using this result we shall study most of the properties of $\mathscr{L}(A)$-invariant subspaces as just properties of ideals of $A$. However in the case of a Malcev algebra $A$, we want to measure the "Lie-ness" of $A$ by considering $A$ as a $\Delta(A, A)$-invariant space. We start by defining a subset of $A$ similar to the nucleus of an alternative algebra defined in [2].

Definition. $N=\{x \in A: x \Delta(A, A)=0\}$ is called the $J$-nucleus of a Malcev algebra $A$; that is, $N$ is the maximal subset of $A$ such that $J(N, A, A)=0$.

Proposition 5.11. If $A$ is a Malcev algebra, then $N J(A, A, A)=0$.

Proof. By (2.14) we have $N J(A, A, A) \subset J\left(N, A, A^{2}\right) \subset J(N, A, A)=0$.

Leмma 5.12. Let $a, b \in A$ be such that $J(a, b, A)=0$, then $a b$ is in $N$.

Proof. By (2.26), we have for $x, y$ in $A, 2 J(a b, x, y)=x J(y, a, b)+J(x, a, b) y$ $-J(x y, a, b)=0$. Therefore by the definition of $N, a b$ is in $N$.

Corollary 5.13. $N$ is an ideal of $A$.

Proof. Clearly $N$ is a linear subspace of $A$ and if $n$ is in $N, a$ in $A$, we have $J(n, a, A)=0$ and by Lemma $5.12, n a$ is in $N$.

In the rest of this section we shall assume that the nonassociative algebra $A$ has no absolute divisors of zero and we can now say that a nonassociative algebra $A$ is simple if and only if $A$ has no proper ideals. This yields $A$ is simple if and only if $A$ is $\mathscr{L}(A)$-irreducible. In the case of a Malcev algebra we have

Theorem 5.14. Let $A$ be a simple Malcev algebra. Then $A=N$ or $A=J(A, A, A)$.

Proof. Since $J(A, A, A)$ is an ideal of $A, J(A, A, A)=A$ or $J(A, A, A)=0$. If $J(A, A, A)=0$, then by Lemma $5.12 A^{2} \subset N$. But since $A$ is simple $A^{2}=A$. Thus $A=N$.

An ideal $B$ of a nonassociative algebra $A$ is called minimal if $B$ contains no proper ideals of $A$ other than $B$ itself. Thus we have that $B$ is a minimal ideal of $A$ if and only if $B$ is a $\mathscr{L}(A)$-irreducible subspace of $A$.

Proposition 5.15. Let $B$ be a minimal ideal of a Malcev algebra $A$. Then (1) $B^{2} \subset N$ or $J(B, B, A)=B$; (2) $B A \subset N$ or $J(B, A, A)=B=J(B, B, A)$.

Proof. (1) Suppose $B^{2} \nsubseteq N$, then by Lemma $5.12 J(B, B, A) \neq 0$. But by Theorem 3.5, $J(B, B, A)$ is an ideal of $A$ contained in $B$ and hence $B=J(B, B, A)$. The proof of (2) is similar.

Recalling that a semi-simple nonassociative algebra $A$ is a direct sum of simple algebras (that are also ideals of $A$ ) and that $\mathscr{L}(A)$ is completely re- 
ducible in $A$ if and only if $A$ is a direct sum of $\mathscr{L}(A)$-irreducible subspaces of $A$, we have $A$ is semi-simple if and only if $\mathscr{L}(A)$ is completely reducible in $A$. For Malcev algebras we have

Lemma 5.16. Let $B$ be an ideal of a semi-simple Malcev algebra $A$ such that $B \cap N=0$. Then $B=J(B, B, B)=J(B, B, A)=J(B, A, A)$.

Proof. The proof is similar to (5.14) and (5.15). It uses the fact that $A=A_{1} \oplus \cdots \oplus A_{k}$ where the $A_{i}$ are simple Malcev algebras such that $A_{i} A_{j}=0$ if $i \neq j$, and furthermore that $B=A_{i_{1}} \oplus \cdots \oplus A_{i_{n}}$.

THEOREM 5.17. If $A$ is a semi-simple Malcev algebra, then $A=N \oplus J(A, A, A)$.

Proof. Since $N$ is an ideal of $A$ and $A$ is semi-simple, we can write $A=N \oplus B$ where $B$ is an ideal of $A$ such that $B \cap N=0$. Then by Lemma 5.16, $B=J(B, A, A) \subset J(A, A, A)$. But $J(A, A, A)=J(N \oplus B, A, A) \subset J(N, A, A)$ $+J(B, A, A)=B$. Hence $B=J(A, A, A)$.

CoROllary 5.18. If $B \neq 0$ is a Lie algebra which is also an ideal in a semisimple Malcev algebra $A$, then $B \subset N$.

Proof. Let $A=A_{1} \oplus \cdots \oplus A_{k}$ and $B=A_{1} \oplus \cdots \oplus A_{r}$ where the $A_{i}$ have the properties given in the proof of Lemma 5.16. Furthermore, since $A_{i}^{2}=A_{i}$, we have $B^{2}=B$. So writing $A=B \oplus C$ where $C$ is again an ideal such that $C B=B C=0$, we have $0=J(B, B, B)=J(B, B, A)$. Therefore by Lemma 5.12, $B=B^{2} \subset N$.

We shall now study the $\Delta(A, A)$-invariant subspaces more closely.

Lemma 5.19. Let $B$ be an ideal of a Malcev algebra $A$, then $\Delta(B, A)$ is an ideal of the Lie algebra $\Delta(A, A)$.

Proof. From (2.38) we have $2(\Delta(x, y), \Delta(u, v))=2 \Delta(x \Delta(u, v), y)$ $+2 \Delta(x, y \Delta(u, v))+6 R_{J(x, y, u v)}-12 \Delta(x y, u v)=2 \Delta(x \Delta(u, v), y)+2 \Delta(x, y \Delta(u, v))$ $+3 \Delta(x, y \cdot u v)+3 \Delta(y, u v \cdot x)+3 \Delta(u v, x y)-12 \Delta(x y, u v)$, by (2.32). Therefore if $x \in B$ and $y, u, v \in A$ we have $(\Delta(x, y), \Delta(u, v)) \in \Delta(B, A)$ and by the linearity of the commutator, $\Delta(B, A)$ is an ideal of $\Delta(A, A)$.

Lemma 5.20. If $B$ is an ideal of $\Delta(A, A)$ and $C$ a $\Delta(A, A)$-invariant subspace of $A$, then $B=C B$ is a $\Delta(A, A)$-invariant subspace of $A$.

Proof. This follows from $(B, \Delta(A, A)) \subset \bigotimes$.

Analogous to Theorem 3.5 we have

Theorem 5.21. If $B$ is an ideal of a Malcev algebra $A$ and $C$ is $a \Delta(A, A)$ invariant subspace of $A$, then $J(A, B, C)$ is a $\Delta(A, A)$-invariant subspace of $A$.

Proof. This follows from the preceding lemmas and from $J(A, B, C)$ $=C \Delta(B, A)$. 
We shall now consider a $\Delta(A, A)$-irreducible subspace of a Malcev algebra A.

TheOREM 5.22. Let $B$ be a $\Delta(A, A)$-irreducible subspace of a Malcev algebra $A$, then $B \subset N$ or $B=J(B, A, A)$.

Proof. Let $C=B \Delta(A, A)$. Then $C$ is a $\Delta(A, A)$-invariant subspace contained in $B$. Therefore $C=0$ or $C=B$.

Now in case $B$ is $\Delta(A, A)$-irreducible and $B C N$, then $\operatorname{dim} B=1$ over the base field $F$. For let $B=e_{1} F \oplus \therefore \oplus e_{r} F \subset N$, then the $e_{i} F$ are subspaces which are $\Delta(A, A)$-invariant. Thus $r=1$. Now again if $B$ is $\Delta(A, A)$-irreducible and $B=J(B, A, A)$, then $B$ is an ideal of $J(A, A, A)$ which contains no proper subspaces that are ideals of $A$. We use these remarks in

THEOREM 5.23. Let $A$ be a Malcev algebra such that $\Delta(A, A)$ is completely reducible in $A$, then $A=N \oplus J(A, A, A)$ where $N$ is completely reduced by $\Delta(A, A)$ and $J(A, A, A)$ is a semi-simple ideal of $A$.

Proof. Since $N$ is a $\Delta(A, A)$-invariant subspace of $A$, write $A=N \oplus B$. We shall show that $B=J(A, A, A)$. Now $B$ is completely reduced by $\Delta(A, A)$, that is, the subspace $B$ is a direct sum of $\Delta(A, A)$-irreducible subspaces, therefore we may write $B=B_{1} \oplus \cdots \oplus B_{r}$ where $B_{i}$ are $\Delta(A, A)$-irreducible subspaces of $A$ such that $B_{i} \Delta(A, A)=B_{i}$, since $B_{i} \cap N=0$. Therefore we have $J(A, A, A)=A \Delta(A, A)=B \Delta(A, A)=\sum_{i} B_{i} \Delta(A, A)=\sum_{i} B_{i}=B$. The rest of the conclusion follows from the remarks preceding the theorem.

Corollary 5.24. If $A$ is a semi-simple Malcev algebra, then $\Delta(A, A)$ is completely reducible in $A$.

We shall finally investigate ideals of $\mathfrak{L}(A)$ and $\Delta(A, A)$ and the subspaces of $A$ induced by these ideals.

Proposition 5.25. Let $B, C$ be ideals of a Malcev algebra $A$, then $(C: B)_{L}$ $=\{T \in \mathscr{L}(A): B T \subset C\}$ is an ideal of $\&(A)$. Also if $B, C$ are $\Delta(A, A)$-invariant subspaces of $A$, then $(C: B)_{\Delta}=\{\Delta \in \Delta(A, A): B \Delta C C\}$ is an ideal of $\Delta(A, A)$.

Proof. This follows easily from the definitions.

Corollary 5.26. Let $B=A E$ be an ideal of $A$, where $E$ is a projection defining $B$. Then $(B: A)_{L}=\{T \in \mathcal{L}(A): T E=T\}=(B: A)_{L} E$. Also if $B=A E$ is a $\Delta(A, A)$-invariant subspace of $A$, where $E$ is a projection defining $B$, then $(B: A)_{\Delta}=\{\Delta \in \Delta(A, A): \Delta E=\Delta\}=(B: A)_{\Delta} E$.

Proof. This also follows from the definitions.

Proposition 5.27. Let $C$ be a $\Delta(A, A)$-invariant subspace of $A$ and let $B$ be an ideal of $\Delta(A, A)$, then $B=C B$ is a $\Delta(A, A)$-invariant subspace of $A$. Also if $C$ is an ideal of $A$ and $B$ is an ideal of $\mathfrak{L}(A)$, then $B$ is an ideal of $A$. 
Note that if $B$ is a $\Delta(A, A)$-invariant subspace of $A$, then by (2.32) $B$ is an ideal of $J(A, A, A)$. Thus the subspace $B$ in the above proposition is an ideal of $J(A, A, A)$.

We shall now investigate the centers of $\mathscr{L}(A)$ and $\Delta(A, A)$. Recall that the center $C$ of a Lie algebra $L$ is the set of all elements $c$ in $L$ such that $[c L]=0$, where $[a b]$ denotes the multiplication of $a$ and $b$ in $L$. Now assume that $A=N \oplus J(A, A, A)$ is a Malcev algebra containing no absolute divisors of zero and that $\mathfrak{e}(\Delta(A, A))$ is the center of the Lie algebra $\Delta(A, A)$. Let $\Delta=\sum_{i} \Delta\left(x_{i}, y_{i}\right) \in \mathfrak{C}(\Delta(A, A))$, then for any $n \in N$ and $x, y, z \in A$ we have $\left(\Delta, R_{n+J(x, y, z)}\right)=\left(\Delta, R_{n}\right)+\left(\Delta, R_{J(x, y, z)}\right)=\left(\Delta, R_{J(x, y, z)}\right)=0$, using (2.32). Thus by linearity we have for any $w \in A=N \oplus J(A, A, A)$ that $0=\left(\Delta, R_{w}\right)$, i.e. $\Delta R_{w}=R_{w} \Delta$. Now let $u, v \in A$, then $u v \Delta=u R_{v} \Delta=u \Delta R_{v}=u \Delta \cdot v$ and $u v \Delta=-v R_{u} \Delta$ $=-v \Delta R_{u}=u \cdot v \Delta$. Also $u v \Delta=u v \sum_{i} \Delta\left(x_{i}, y_{i}\right)=\sum_{i} J\left(u v, x_{i}, y_{i}\right)$ and obtain, using (2.26), that $u v \Delta=\sum_{i}\left[u J\left(v, x_{i}, y_{i}\right)+J\left(u, x_{i}, y_{i}\right) v-2 J\left(x_{i} y_{i}, u, v\right)\right]$ $=\sum_{i}\left[u \cdot v \Delta\left(x_{i}, y_{i}\right)+u \Delta\left(x_{i}, y_{i}\right) \cdot v-2 J\left(x_{i} y_{i}, u, v\right)\right]=u \cdot v \Delta+u \Delta \cdot v-2 J(z, u, v)$, where $z=\sum_{i} x_{i} y_{i}$. Thus $u v \Delta=2 u v \Delta-2 J(z, u, v)$, by the preceding remarks, and therefore $u v \Delta=2 J(z, u, v)$.

CASE 1. If $z=0$, then for all $u, v$ in $A, 0=u v \Delta=u \Delta \cdot v$. Now if $\Delta \neq 0$, then there exists $u_{0} \in A$ such that $u_{0} \Delta \neq 0$. But for all $v \in A$ we have $0=u_{0} \Delta \cdot v$ and since there are no absolute divisors of zero we obtain $u_{0} \Delta=0$, a contradiction. Therefore $z=0$ implies $\Delta=0$, i.e., $\Delta \neq 0$ implies $z \neq 0$.

CASE 2. If $z \neq 0$, then again since there are no absolute divisors of zero in $A$ there exists $z_{0} \in A$ such that $a=z z_{0} \neq 0$. Therefore $a \Delta=z z_{0} \Delta=2 J\left(z, z, z_{0}\right)=0$ and hence $a$ is in the null space of $\Delta$. Combining these cases we have

Lemma 5.28. If $A=N \oplus J(A, A, A)$ is a Malcev algebra containing no absolute divisors of zero and if $0 \neq \Delta \in \mathrm{e}(\Delta(A, A))$, the center of $\Delta(A, A)$, then $\Delta$ is singular.

Now we may write $\operatorname{Ker}(\Delta)$ for the null space of the linear transformation $\Delta$ and show

Lemma 5.29. If $0 \neq \Delta \in \mathbb{e}(\Delta(A, A))$ and $A=N \oplus J(A, A, A)$ has no absolute divisors of zero, then $A \Delta$ and $\operatorname{Ker}(\Delta)$ are proper ideals of $A$.

Proof. Since $\Delta \neq 0, z \neq 0$ and therefore $a=z z_{0} \in \operatorname{Ker}(\Delta)$. Thus $A \Delta$ is a proper subspace of $A$. Now for any $u \in A$ and $v \Delta \in A \Delta$, we have $v \Delta \cdot u=v u \cdot \Delta \in A \Delta$. Thus $A \Delta$ is an ideal of $A$. Since $\Delta \neq 0, \operatorname{Ker}(\Delta)$ is a proper subspace, by the preceding lemma. Now for any $u \in \operatorname{Ker}(\Delta)$ and $v \in A$ we have $u v \Delta=u \Delta \cdot v=0$ and therefore $\operatorname{Ker}(\Delta)$ is an ideal of $A$.

Corollary 5.30. If $A$ is $\Delta(A, A)$-irreducible, then $\mathfrak{e}(\Delta(A, A))=0$.

Proof. On one hand, $A=N$ and therefore $\mathfrak{e}(\Delta(A, A)) \subset \Delta(A, A)=0$. Otherwise $A=J(A, A, A)$ and $N=0$ so that $A=N \oplus J(A, A, A)$. But if $0 \neq \Delta$ $\in \mathfrak{C}(\Delta(A, A))$, then $A \Delta$ and $\operatorname{Ker}(\Delta)$ are proper ideals of $A$ and therefore 
proper $\Delta(A, A)$-invariant subspaces of $A$. Hence we must have $\mathfrak{e}(\Delta(A, A))$ $=0$.

Corollary 5.31. If $\Delta(A, A)$ is completely reducible in $A$, then $\mathfrak{e}(\Delta(A, A))$ $=0$.

Proof. This follows from $A=B_{1} \oplus \cdots \oplus B_{n}$ where $B_{i}$ are $\Delta(A, A)$ irreducible and $\Delta(A, A)=\sum_{i} \Delta\left(B_{i}, B_{i}\right)$ and hence $\mathfrak{e}(\Delta(A, A))$ $=\bigcap_{i} \mathfrak{e}\left(\Delta\left(B_{i}, B_{i}\right)\right)$.

COROLlaRy 5.32. If $A$ is a semi-simple Malcev algebra, then $\mathfrak{C}(\mathscr{L}(A))$, the center of the Lie transformation algebra, is zero.

Proof. Since $A$ is semi-simple, $A=N \oplus J(A, A, A)$ and $A$ has no absolute divisors of zero. An easy calculation shows $\mathcal{L}(A)=R(N) \oplus \Delta(A, A)$ and $\mathfrak{e}(\mathfrak{L}(A))=\mathfrak{e}(R(N)) \oplus \mathfrak{e}(\Delta(A, A))$. Now since $A$ is semi-simple, $\Delta(A, A)$ is completely reducible in $A$ (Corollary 5.24) and by the preceding corollary $\mathfrak{e}(\Delta(A, A))=0$. Now let $R(C)=\mathfrak{C}(R(N))$, the center of the Lie algebra $R(N)$, where $C \subset N$. Then $0=(R(C), R(N))=R(C N)$, since $\Delta(N, N)=0$. Therefore since there are no absolute divisors of zero in $A, C N=0$. But $A C=N C+J(A, A, A) C=0$, since $C \subset N$ and $J(A, A, A) N=0$. This implies $C=0$.

6. $J$-potency. In this section we shall consider a concept similar to solvability which is useful in the discussion of the radical of a Malcev algebra.

Definition. Let $B$ be an ideal in a Malcev algebra $A$.

$$
\begin{aligned}
& \text { Set } J_{0}(B)=J(B, B, B) \quad \text { and } \quad J_{0}^{k+1}(B)=J\left(J_{0}^{k}(B), J_{0}^{k}(B), J_{0}^{k}(B)\right) . \\
& \text { Set } J_{1}(B)=J(B, B, A) \quad \text { and } \quad J_{1}^{k+1}(B)=J\left(J_{1}^{k}(B), J_{1}^{k}(B), A\right) . \\
& \text { Set } J_{2}(B)=J(B, A, A) \quad \text { and } \quad J_{2}^{k+1}(B)=J\left(J_{2}^{k}(B), A, A\right) .
\end{aligned}
$$

An ideal $B$ of $A$ is called $J_{i}$-potent, $i=0,1,2$, if there exists an integer $N \geqq 2$ such that $J_{i}^{N}(B)=0$.

Remarks. (1) The case $N=1$ is excluded because this case causes some difficulty with the $J$-nucleus. (2) For all $n$ we have $J_{0}^{n}(B) \subset J_{1}^{n}(B) \subset J_{2}^{n}(B)$. (3) For $i=0,1,2$ we have $B \supset J_{i}(B) \supset J_{i}^{2}(B) \supset \cdots$. (4) A $J_{0}$-potent ideal will simply be called $J$-potent.

We shall now obtain several conditions for an ideal of a Malcev algebra to be $J$-potent. Thus let $B$ be an ideal of a Malcev algebra $A$ and let $\Delta(x, y)$ $=\left(R_{x}, R_{y}\right)-R_{x y}$, where $z \Delta(x, y)=J(z, x, y)$ (see (2.27)). Now defining $J^{0}(B)=B$, we have $\zeta \in J(B)$ if and only if $\zeta=\sum_{i} J\left(\beta_{i}, b_{1 i}, b_{2 i}\right)=\sum_{i} \beta_{i} \Delta\left(b_{1 i}, b_{2 i}\right)$, where $\beta_{i}, b_{1 i}, b_{2 i}$ are in $J^{0}(B)$; that is, $\zeta \in J(B)$ if and only if $\zeta \in B \Delta(B, B)$. Similarly $\zeta \in J^{2}(B)$ if and only if $\zeta=\sum_{i} J\left(j_{i}, j_{1 i}, j_{2 i}\right)$, where $j_{i}, j_{1 i}, j_{2 i}$ are in $J(B)$. Thus

$$
\zeta=\sum_{i} j_{i} \Delta\left(j_{1 i}, j_{2 i}\right)=\sum_{i} \sum_{k} \beta_{i k} \Delta\left(b_{1 i k}, b_{2 i k}\right) \Delta\left(j_{1 i}, j_{2 i}\right) ;
$$


that is, $\zeta \in J^{2}(B)$ if and only if $\zeta \in B \Delta(B, B) \Delta(J(B), J(B))$. By induction, we obtain $\zeta \in J^{k+1}(B)$ if and only if $\zeta \in B \Delta(B, B) \cdots \Delta\left(J^{k}(B), J^{k}(B)\right)$. Thus $J^{k+1}(B)=B \prod_{i=0}^{k} \Delta\left(J^{i}(B), J^{i}(B)\right)$, where this is an ordered product.

Now since the descending chain condition for the subspaces $\Delta\left(J^{i}(B), J^{i}(B)\right)$ holds, we can assume that there exists an integer $N$ such that $\Delta\left(J^{N}(B), J^{N}(B)\right)$ $=\Delta\left(J^{N+1}(B), J^{N+1}(B)\right)=\cdots$. Calling the minimal such integer the index of $B$ provided that $N \geqq 1$, we have

TheORem 6.1. An ideal $B$ of a Malcev algebra $A$ is J-potent if and only if the index $N$ of $B$ is such that $B \prod_{i=0}^{N} \Delta\left(J^{i}(B), J^{i}(B)\right)=0$.

Corollary 6.2. An ideal $B$ of $A$ is $J$-potent if the index $N$ of $B$ is such that every element of $\Delta\left(J^{N}(B), J^{N}(B)\right)$ is associatively nilpotent.

Proof. By the definition of the index of $B$,

$$
J^{N+1}(B)=B \prod_{i=0}^{N} \Delta\left(J^{i}(B), J^{i}(B)\right)=B \prod_{i=0}^{\infty} \Delta\left(J^{i}(B), J^{i}(B)\right) .
$$

By (2.14) and induction we obtain for every integer $n, J^{n}(B) J^{n}(B) \subset J^{n}(B)$ and therefore by (2.38) and the above remark (3), $\Delta\left(J^{N}(B), J^{N}(B)\right)$ is closed under commutation. Now using Theorem 1, page 201 of [3] concerning weakly closed sets, we see that the enveloping algebra of $\Delta\left(J^{N}(B), J^{N}(B)\right)$ is an associative nilpotent algebra of linear transformations and hence for large enough $M, \prod_{i=N}^{M} \Delta\left(J^{i}(B), J^{i}(B)\right)=0$. Thus $B$ is $J$-potent.

We now consider a concept similar to the maximal solvable ideal of an algebra.

Lemмa 6.3. If $U, V$ are $J$-potent ideals of a Malcev algebra $A$, then so is $U+V$.

Proof. First note that for $E, F$ subsets of the ideals $U, V$ and $D \subset A$ we have $J(E, F, D) \subset U \cap V$. Then $J^{1}(U+V) \subset J(U, U+V, U+V)$ $+J(V, U+V, U+V) \subset J(U, U, U+V)+J(U, V, U+V)+J(V, U, U+V)$ $+J(V, V, U+V) \subset J(U, U, U)+J(U, U, V)+J(V, V, U)+J(V, V, V)$ $+U \cap V \subset J^{1}(U)+J^{1}(V)+U \cap V$. Similarly we can show by induction that for every integer $n, J^{n}(U+V) \subset J^{n}(U)+J^{n}(V)+U \cap V$. Therefore for large enough $N, J^{N}(U+V) \subset U \cap V \subset U$ and for a larger $M, J^{M}(U+V) \subset J^{M}(U)$ $=0$.

We can now define a unique maximal $J$-potent ideal of $A: \Im(A)=\sum_{\alpha} U_{\alpha}$ where the $U_{\alpha}$ are $J$-potent ideals of $A$. Similarly there exist unique maximal $J_{i}$-potent ideals $\Im_{1}(A)$ and $\Im_{2}(A)$ of $A$.

Proposition 6.4. Let $\Im(A)$ be the maximal J-potent ideal of a Malcev algebra $A$. Then (1) $\Im(A / \Im(A))=0$; (2) if $B$ is an ideal of $A$ such that $\Im(A / B)$ $=0$, then $\Im(A) \subset B$ or $J(\Im(A)) \subset B$. 
Proof. (1) If $\Im(A / \Im(A)) \neq 0$, then there is an ideal $B$ of $A$ such that $\bar{B}=B+\Im(A)$ is a nonzero $J$-potent ideal of $A / \Im(A)$. But this means $J^{N}(B)$ $\subset \Im(A)$ for large enough $N$ and hence $J^{M}(B)=0$ for a still larger $M$. Therefore $B \subset \Im(A)$, contradicting the assumption that $\bar{B}$ is a nonzero ideal of $A / \Im(A)$. (2) The coset $\bar{F}=\Im(A)+B$ is such that if $J(\bar{F}) \neq \overline{0}$, i.e., $J(\Im(A)) \nsubseteq B$, then $\bar{F}$ is $J$-potent in $A / B$ and therefore $\bar{F}=\overline{0}$, i.e., $\Im(A) \subset B$. Similar statements hold for the maximal $J_{i}$-potent ideals $i=1,2$.

TheORem 6.5. Let $S$ be the maximal solvable ideal of a Malcev algebra $A$. Then (1) $\Im_{2}(A) \subset \Im_{1}(A) \subset \Im(A)$; (2) $\Im_{1}(A) \cap S \neq 0$ if $\Im_{1}(A) \neq 0$; (3) $S=0 \mathrm{im}$ plies $\Im_{1}(A)=0$; (4) $S \subset \Im(A)$ if $S$ is not a Lie algebra.

Proof. (1) From the remark (2) following the definition of $J_{i}$-potency, we have that $\Im_{2}^{N}(B)=0$ implies $\Im_{1}^{N}(B)=0$ implies $\Im^{N}(B)=0$. Thus by the definition of $\Im_{i}(A)$, (1) follows. (2) Let $J_{1}^{N+1}(B)=J\left(J_{1}^{N}(B), J_{1}^{N}(B), A\right)=0$, where $C=J_{1}^{N}(B) \neq 0$ and $B$ is an ideal of $A$. Then $C$ is an ideal of $A$. Certainly $J_{1}(B)=J(B, B, A)$ is an ideal of $A$ and if we assume $J_{1}^{K}(B)$ is an ideal of $A$, then $J_{1}^{K+1}(B)=J\left(J_{1}^{K}(B), J_{1}^{K}(B), A\right)$ is an ideal of $A$ by Theorem 3.5. Now $J(C, C, A)=0$, therefore by Lemma $5.12, C^{2} \subset N$. But also $C \subset J(A)$ and $C^{2} \subset J(A)$. Hence by Proposition 5.11, $C^{2} \cdot C^{2} \subset N J(A)=0$ and $C$ is solvable. Thus if $\Im_{1}(A) \neq 0,0 \neq C \subset B \subset \Im_{1}(A)$ and $C \subset S$ so therefore $C \subset \Im_{1}(A) \cap S$. (3) If $S=0$, then $J_{1}(A) \cap S=0$ and therefore using the contrapositive of (2), $J_{1}(A)=0$. (4) Let $B$ be a non-Lie ideal of $A$, then $J(B) \subset B^{(2)}=B \cdot B \neq 0$ and by induction, $J^{K+1}(B) \subset B^{(K+1)} \cdot B^{(K+1)}=B^{(K+2)}$. Thus if $B$ is solvable, then $B$ is $J$-potent.

TheOREm 6.6. Let $A$ be a semi-simple, non-Lie Malcev algebra, then $\Im(A)$ $=0$.

Proof. Since $A$ is semi-simple write $\Im(A)=N_{1} \oplus B_{1} \oplus \cdots \oplus B_{n}$ where $N_{1} \subset N$ and the $B_{i}$ are simple subalgebras of $J(A)$ such that $B_{i} B_{j}=0$ if $i \neq j$. Then

$$
J(\Im(A))=\sum_{i, j, k} J\left(B_{i}, B_{j}, B_{k}\right)=\sum_{k} J\left(B_{k}\right)=\sum_{k} B_{k}
$$

and by induction $J^{n}(\Im(A))=\sum_{k} B_{k}$. Since $\Im(A)$ is $J$-potent, $\sum_{k} B_{k}=0$. But then $\Im(A)=N_{1}$ and therefore $J(\Im(A))=0$. However by definition we must have an integer $N \geqq 2$ such that $J^{N}(\Im(A))=0$.

Analogous to $J$-potent ideals we define a $\Delta(A, A)$-invariant subspace $B$ of $A$ to be $J$-potent if defining $J^{1}(B)=J(B, B, B)=B \Delta(B, B) \subset B$ and $J^{k+1}(B)=J^{k}(B) \Delta\left(J^{k}(B), J^{k}(B)\right) \subset J^{k}(B)$, there exists an integer $N \geqq 2$ such that $J^{N}(B)=0$.

In a manner similar to that for ideals, we obtain a unique maximal $J$ potent $\Delta(A, A)$-invariant subspace, $\Im(\Delta, A)$, of $A$.

Theorem 6.7. If $\Delta(A, A)$ is completely reducible in $A$, then $\Im(\Delta, A)=0$. 
Proof. Similar to Theorem 6.6.

Proposition 6.8. Let $B$ be an ideal of $A$ such that $\bar{A}=A / B$ is completely reduced by $\Delta(\bar{A}, \bar{A})$, then $\Im(\Delta, A) \subset B$ or $J(\Im(\Delta, A)) \subset B$.

Proof. Using Theorem 6.7, the proof is similar to that of Proposition 6.4.

Proposition 6.9. $\Im(A) \subset \Im(\Delta, A)$.

Proof. $\Im(A)$ is a $J$-potent ideal and therefore a $J$-potent $\Delta(A, A)$-invariant subspace of $A$.

We shall use these results in determining some properties of the radical of a Malcev algebra.

7. The radical of a Malcev algebra. The radical of an arbitrary finite dimensional nonassociative algebra $A$ is defined by Albert in [1] to be the minimal ideal $\mathfrak{N}$ (sometimes denoted by $\operatorname{rad}(A)$ ) of $A$ such that $A / \mathfrak{N}$ is zero or semi-simple, that is, a direct sum of simple subalgebras. Furthermore in [1] it is shown that if $A$ is homomorphic to a semi-simple algebra, then the radical, $\mathfrak{N}$, exists in the sense that $A / \mathfrak{N}$ is semi-simple and $\mathfrak{N}$ is contained in every ideal $B$ of $A$ for which $A / B$ is semi-simple. We shall now investigate the relationships between the Lie transformation algebra, $\mathscr{L}(A)$, and the radical $\mathfrak{N}$ of $A$. For this we need the following theorem of Jacobson [4]:

THEOREM 7.1. Let $L$ be a Lie algebra of linear transformations in a finite dimensional vector space $M$ over a field of characteristic zero. Then $L$ is completely reducible in $M$ if and only if the following conditions hold: (1) $L=C \oplus L_{1}$, where $L_{1}$ is a semi-simple ideal of $L$ and $C$ the center of $L$ and (2) the elements of $C$ are semi-simple. An element of $C$ is semi-simple means that its minimum polynomial is a product of distinct irreducible polynomials.

Again we shall assume for the rest of this section that the nonassociative algebra $A$ has no absolute divisors of zero. Using Theorem 7.1 we have

THEOREM 7.2. Let $A$ be a nonassociative algebra over a field of characteristic zero. Then $A$ is semi-simple if and only if $\mathscr{L}(A)$ is completely reducible in $A$ if and only if $\mathfrak{L}(A)=\mathfrak{C}(\mathfrak{L}(A)) \oplus \mathfrak{L}_{1}(A)$ where $\mathfrak{L}_{1}(A)$ is a semi-simple ideal of $\mathfrak{L}(A)$ and $\mathfrak{C}(\mathscr{L}(A))$ is the center of $\mathfrak{L}(A)$ which consists of semi-simple elements.

Corollary 7.3. Let $A$ be a nonassociative algebra over a field of characteristic zero such that $A$ being semi-simple implies $\mathcal{C}(\mathcal{L}(A))=0$. Then $A$ is semisimple if and only if $\mathcal{L}(A)$ is a semi-simple Lie algebra. (Note that from Corollary 5.32 a Malcev algebra has this property.)

THEOREM 7.4. Let $B$ be an ideal of a nonassociative algebra $A$, then $\&(A / B)$ $\cong \mathscr{L}(A) /(B: A)_{L}$ where $(B: A)_{L}=\{T \in \mathfrak{L}(A): A T \subset B\}=\{T \in \mathfrak{L}(A): T E=T\}$ and $E$ is a projection defining $B=A E$.

Proof. It suffices to show that if $T=\tau\left(x_{1}, \cdots, x_{n}\right) \in \mathfrak{L}(A)$, then the map 
$\eta: T \rightarrow \tau\left(\bar{x}_{1}, \cdots, \bar{x}_{n}\right)$ of $\mathscr{L}(A)$ onto $\mathscr{L}(A / B)$, where $\bar{x}_{i} \in A / B=\bar{A}$, coincides with the induced map $\bar{\eta}: T \rightarrow \bar{T}$ of $\mathscr{L}(A)$ onto $\overline{\mathscr{L}}(A)$ where $\bar{T} \in \overline{\mathfrak{L}}(A)$ is defined by $\bar{a} \cdot \bar{T}=[a T]-$. For suppose $\bar{T}=\tau\left(\bar{x}_{1}, \cdots, \bar{x}_{n}\right)$, then from Chapter $4, \S 2$ of [5] we see that the map $\bar{\eta}: T \rightarrow \bar{T}$ is a homomorphism of $\mathscr{L}(A)$ onto $\mathscr{L}(A / B)$ and that the kernel of $\bar{\eta}$ is $(B: A)_{L}$. The rest of the theorem now follows. Now to show $\bar{T}=\tau\left(\bar{x}_{1}, \cdots, \bar{x}_{n}\right): \bar{a} \bar{T}=[a T]-=\left[a \tau\left(x_{1}, \cdots, x_{n}\right)\right]^{-}$ $=\bar{a} \tau\left(\bar{x}_{1}, \cdots, \bar{x}_{n}\right)$, because $\tau$ is a polynomial in $R_{x_{i}}$ and $L_{x_{j}}$ and using an extension of $\left[a R_{x_{i}}\right]^{-}=\bar{a} \bar{x}_{i}$ and $\left[a L_{x_{j}}\right]^{-}=\bar{a} \bar{x}_{j}$. Thus $\bar{T}=\tau\left(\bar{x}_{1}, \cdots, \bar{x}_{n}\right)$.

Corollary 7.5. If $A$ is a nonassociative algebra and $B$ is an ideal of $\mathscr{L}(A)$, then $\mathfrak{L}(A / A @) \cong(\mathscr{L}(A) / B) /\left((A ß: A)_{L} / B\right)$.

Proof. From Proposition 5.27, $A B$ is an ideal of $A$. The isomorphism follows from the second isomorphism theorem noting that $B \subset(A B: A)_{L}$.

THEOREM 7.6. Let $A$ be a nonassociative algebra over a field of characteristic zero such that $A$ is properly homomorphic to a semi-simple algebra. Assume if $B$ is an ideal of $A$ such that $A / B$ is semi-simple, then $\mathcal{C}(\mathcal{L}(A / B))=0$. Then the radical $\mathfrak{N}$ of $A$ equals $A \mathcal{S}$ where $S$ is the radical of $\mathfrak{L}(A)$.

Proof. Since $A$ is homomorphic to a semi-simple algebra, let $B$ be an ideal of $A$ such that $A / B$ is semi-simple. Then $\mathscr{L}(A / B) \cong \mathscr{L}(A) /(B: A)_{L}$ is a semisimple Lie algebra and therefore $\delta \subset(B: A)_{L}$. Now since $B$ is an ideal, $B=A E$ where $E$ is a projection and it is easily seen that $(B: A)_{L}=(B: A)_{L} E$. Therefore $A S \subset A(B: A)_{L}=A(B: A)_{L} E \subset A E=B$. In particular this shows $A S$ is a proper ideal of $A$ if $\$ \neq 0$ and when $B=\mathfrak{N}$ we obtain $A S \subset \mathfrak{N}$. To obtain the reverse inclusion note that $\mathscr{L}\left(A / A \mathcal{S} \cong(\mathscr{L}(A) / \mathfrak{S}) /\left((A \mathcal{S}: A)_{L} / \mathfrak{S}\right)\right.$ and therefore $\mathscr{L}(A / A S)$ is semi-simple. By Theorem 7.2 with $\mathfrak{C}(\mathscr{L}(A / A \mathcal{S}))=0$ we obtain $A / A S$ is semi-simple and hence $\mathfrak{N} \subset A S$.

CoROllaRy 7.7. Let $A$ be a Malcev algebra over a field of characteristic zero and let $A$ be properly homomorphic to a semi-simple algebra, then the radical of $A$ is $A \mathcal{S}$ where $\mathcal{S}$ is the radical of the Lie algebra $\mathfrak{L}(A)$.

Proof. This follows from Theorem 7.6 and Corollary 5.32.

We shall now investigate the subspace $A \sigma$, where $\sigma$ is the radical of $\Delta(A, A)$, and find relationships between this subspace and the radical $A S$. One difficulty that arises is the investigation of $\Delta(\bar{A}, \bar{A})$ where $\bar{A}=A / B$ with $B$ being a $\Delta(A, A)$-invariant subspace of $A: \Delta(x, y)$ is defined on an algebra and multiplication is involved, but $\bar{A}$ need not be an algebra. To circumvent this difficulty we shall show that the map $\bar{\Delta}(x, y)$ induced by $\Delta(x, y)$ on $\bar{A}$ can be identified in a natural way with $\Delta(\bar{x}, \bar{y})$. To do this first consider $[J(x, y, z)]^{-}$and $J(\bar{x}, \bar{y}, \bar{z})$ as subsets of $A$. Since $\bar{A}$ need not be an algebra, $J(\bar{x}, \bar{y}, \bar{z})$ need not be defined in terms of products, but since $B$ is a $\Delta(A, A)$ invariant subspace of $A, J(\bar{x}, \bar{y}, \bar{z})$ can be identified with $[J(x, y, z)]^{-}$ in $\bar{A}=A / B$. For $J(\bar{x}, \bar{y}, \bar{z})=J(x+B, y+B, z+B) \subset J(x, y, z)+B=[J(x, y, z)]^{-}$, 
since $B$ is a $\Delta(A, A)$-invariant subspace of $A$. Now if $J(\bar{x}, \bar{y}, \bar{z})=J(\bar{u}, \bar{v}, \bar{w})$, then $J(\bar{x}, \bar{y}, \bar{z}) \subset[J(u, v, w)]-$ and $J(\bar{u}, \bar{v}, \bar{w}) \subset[J(x, y, z)]-$. Thus the identification of $J(\bar{x}, \bar{y}, \bar{z})$ with $[J(x, y, z)]-$ in $A / B$ is single-valued. Now let $\bar{\Delta}(x, y)$ be the linear transformation induced on $\bar{A}=A / B$ by $\Delta(x, y)$, i.e., $\bar{z} \bar{\Delta}(x, y)$ $=[z \Delta(x, y)]^{-}$and let $\bar{\Delta}(A, A)$ be the linear space spanned by these transformations. Furthermore let $\Delta(\bar{A}, \bar{A})$ be the linear space spanned by the transformations $\Delta(\bar{x}, \bar{y})$ defined by $\bar{z} \Delta(\bar{x}, \bar{y})=J(\bar{z}, \bar{x}, \bar{y})$, then we have by the various definitions, $\bar{z} \bar{\Delta}(x, y)=[z \Delta(x, y)]^{-}=[J(z, x, y)]^{-}=J(\bar{z}, \bar{x}, \bar{y})=\bar{z} \Delta(\bar{x}, \bar{y})$ and hence $\bar{\Delta}(x, y)=\Delta(\bar{x}, \bar{y})$. By linearity we obtain $\bar{\Delta}(A, A)=\Delta(\bar{A}, \bar{A})$. As an application we have

Proposition 7.8. Let $B$ be a $\Delta(A, A)$-invariant subspace of $A$ and $\bar{A}=A / B$. Then $\Delta(\bar{A}, \bar{A})$ is a Lie algebra and $\Delta(\bar{A}, \bar{A}) \cong \Delta(A, A) /(B: A)_{\Delta}$ as Lie algebras.

Proof. Let $\tau: \Delta(x, y) \rightarrow \bar{\Delta}(x, y)$ and extend $\tau$ in a linear fashion to a map $T: \Delta(A, A) \rightarrow \bar{\Delta}(A, A)$. It is easily seen that $T$ is a homomorphism of the Lie algebra $\Delta(A, A)$ onto the Lie algebra $\bar{\Delta}(A, A)$. But $\bar{\Delta}(A, A)=\Delta(\bar{A}, \bar{A})$. Thus $\Delta(\bar{A}, \bar{A})$ is a Lie algebra and the kernel of $T$ is clearly $(B: A)_{\Delta}$.

Corollary 7.9. Let $B$ be an ideal of the Lie algebra $\Delta(A, A)$ and let $B=A \otimes$, then $\Delta(A / B, A / B) \cong(\Delta(A, A) / B) /\left((B: A)_{\Delta} / B\right)$.

TheOREM 7.10. Let $A$ be a Malcev algebra over a field of characteristic zero, then $\Delta(A, A)$ is completely reducible in $A$ if and only if $\Delta(A, A)$ is a semi-simple Lie algebra.

Proof. The proof is similar to that of Corollary 7.3, just noting that $\Delta(A, A)$ being completely reducible in $A$ implies $\mathrm{e}(\Delta(A, A))=0$.

We shall now investigate the minimal $\Delta(A, A)$-invariant subspace, $\mathfrak{M}$, of $A$ such that $\bar{A}=A / \mathfrak{M}$ is completely reduced by $\Delta(\bar{A}, \bar{A})$. Assume that there exists a $\Delta(A, A)$-invariant subspace $B$ of $A$ such that $\bar{A}=A / B$ is completely reduced by $\Delta(\bar{A}, \bar{A})$, then by the minimum condition for $\Delta(A, A)$ invariant subspaces of $A$ there exists a minimal such subspace, $\mathfrak{M}$, of $A$. We now have

THEOREM 7.11. Let $A$ be a Malcev algebra over a field of characteristic zero and let $B$ be a proper $\Delta(A, A)$-invariant subspace of $A$ such that $\bar{A}=A / B$ is completely reduced by $\Delta(\bar{A}, \bar{A})$. Then $\mathfrak{M}=A \sigma$ where $\sigma$ is the radical of the Lie algebra $\Delta(A, A)$.

Proof. Similar to Theorem 7.6.

We shall eventually show $A \sigma$ is an ideal of $A$, but first we have

Lemma 7.12. If $B$ is a $\Delta(A, A)$-invariant subspace of a Malcev algebra $A$ such that $B$ is properly contained in $J(A)$ and $\bar{A}=A / B$ is completely reduced by $\Delta(\bar{A}, \bar{A})$, then a nontrivial multiplication can be defined on $\bar{A}$ so that it becomes a Malcev algebra. 
Proof. Since $\bar{A}$ is completely reduced by $\Delta(\bar{A}, \bar{A}), \bar{A}=\bar{N} \oplus J(\bar{A})$. We shall first define a multiplication for $\bar{N}=\{\bar{x}=x+B: \bar{x} \Delta(\bar{A}, \bar{A})=\overline{0}\}$. We have $\overline{0}=\bar{N} \Delta(\bar{A}, \bar{A})=J(\bar{N}, \bar{A}, \bar{A})=[J(M, A, A)]-$ where $M$ is a subset of $A$ such that $\bar{N}=M / B$. Thus we have $J(M, A, A) \subset B$, i.e., if $\bar{x}=x+B \in \bar{N}$, then $J(x, A, A)=x \Delta(A, A) \subset B$. Now let $\bar{n}_{1}, \bar{n}_{2} \in \bar{N}$ and set $\bar{n}_{1} \bar{n}_{2}=\left[n_{1} n_{2}\right]^{-}$. This is well defined, for if $\bar{n}_{1}=\bar{m}_{1}, \bar{n}_{2}=\bar{m}_{2}$, then $n_{1}-m_{1} \in B$ and $n_{2}-m_{2} \in B$ and therefore $n_{1} n_{2}-m_{1} n_{2} \in n_{2} B \subset n_{2} J(A)=n_{2} R(J(A)) \subset n_{2} \Delta(A, A) \subset B$, by the preceding remarks where $R(J(A))$ denotes right multiplication by $J(A)$. Also $m_{1} n_{2}-m_{1} m_{2}$ $\in B$ and adding these results we obtain $\left[n_{1} n_{2}\right]^{-}=\left[m_{1} m_{2}\right]^{-}$. Thus the multiplication is well defined and using $J(M, A, A) \subset B$ where $\bar{N}=M / B$ together with (2.26) we obtain $\bar{n}_{1} \bar{n}_{2}=\left[n_{1} n_{2}\right]-\in \bar{N}$. Thus $\bar{N}$ is an algebra. Now to define multiplication between $\bar{N}$ and $J(\bar{A})=\bar{A} \Delta(\bar{A}, \bar{A})$. For $\bar{n} \in \bar{N}$ and $j \in J(\bar{A})$, set $\bar{n} \bar{j}=[n j]^{-}$. This is also well defined, for if $\bar{n}_{1}=\bar{n}_{2}, \bar{j}_{1}=\bar{j}_{2}$, then $n_{1}-n_{2} \in B$ and $j_{1}-j_{2} \in B$. Now we can assume $j_{i} \in J(A)$, since $B \subset J(A)$ and $j_{i} \in J(A)+B$. Therefore $n_{1} j_{1}-n_{1} j_{2} \in n_{1} B \subset B$, as before. Also $n_{1} j_{2}-n_{2} j_{2} \in B j_{2} \subset B \Delta(A, A) \subset B$ and adding we obtain $\left[n_{1} j_{1}\right]^{-}=\left[n_{2} j_{2}\right]^{-}$and the multiplication between $\bar{N}$ and $J(\bar{A})$ is well defined. Note that $\bar{n} \bar{j}=[n j]-\in[N \Delta(A, A)]^{-} \subset \bar{B}=\overline{0}$ and thus $\bar{N} J(\bar{A})=\overline{0}$. In a similar way we define for $j_{i} \in J(\bar{A}), j_{1} \bar{j}_{2}=\left[j_{1} j_{2}\right]-$. It is clear that $\bar{A}$ is now a Malcev algebra by extending the multiplication to all of $\bar{A}$ via the distributive laws. The multiplication is nontrivial, for if $\bar{A}^{2}=\overline{0}$ then we see $J(\bar{A}) \subset \bar{A}^{2}=\overline{0}$ and therefore $J(A) \subset B$. So $B=J(A)$, contrary to the hypothesis that $B \neq J(A)$.

We shall always consider a quotient space $\bar{A}$ satisfying this theorem as a Malcev algebra with multiplication $\bar{a}_{1} \bar{a}_{2}=\left[a_{1} a_{2}\right]^{-}$where $\bar{a}_{i} \in A / B=\bar{A}$. Furthermore it is easily seen that the map $\eta: a \rightarrow \bar{a}=a+B$ is an algebra homomorphism of $A$ onto the Malcev algebra $\bar{A}$. Let $B^{\prime}$ be the kernel of this algebra homomorphism, then $B^{\prime}$ is an ideal of $A$ and clearly $B^{\prime}=B$. This proves

THEOREM 7.13. If $B \subset J(A)$ is a $\Delta(A, A)$-invariant subspace of a Malcev algebra $A$ such that $\bar{A}=A / B$ is completely reduced by $\Delta(\bar{A}, \bar{A})$, then $B$ is an ideal of $A$.

Proof. The case $B=J(A)$ is clear because $B$ is already an ideal.

Corollary 7.14. $\bar{A}=A / A \sigma$ is a Malcev algebra and $A \sigma$ is an ideal of $A$.

Now we investigate relationships between $A S$ and $A \sigma$ in the following

TheOREM 7.15. Let A be a Malcev algebra over a field of characteristic zero and let $\mathfrak{N}=A \mathcal{S}, \mathfrak{R}=A \sigma$ where $\mathcal{S}(\sigma)$ is the radical of the Lie algebra $\mathfrak{L}(A)$ $(\Delta(A, A))$. Then

(1) $\mathfrak{N} / \mathfrak{M}$ is the radical of $\bar{N}$ where $\bar{N}$ is the $J$-nucleus of $\bar{A}=A / \mathfrak{M}$.

(2) There exists an integer $k$ such that $\mathfrak{R}^{(k)} \subset \mathfrak{M}$ and $\mathfrak{N} \Delta(A, A) \subset \mathfrak{M}$.

(3) $A / \mathfrak{M} \cong \mathfrak{N} / \mathfrak{M} \oplus A / \mathfrak{N}$ where $A / \mathfrak{N}(\mathfrak{N} / \mathfrak{M})$ is a subalgebra (ideal) of $\mathfrak{R} / \mathfrak{M} \oplus A / \mathfrak{R}$. 
(4) $\mathscr{L}(\mathfrak{N} / \mathfrak{M})$ is the radical of the Lie algebra $\mathscr{L}(A / \mathfrak{M})$.

(5) $(\mathcal{S}, \Delta(A, A)) \subset \sigma$.

Proofs. (1). First we note that if $B \subset J(A)$ is a $\Delta(A, A)$-invariant subspace of $A$ such that $\bar{A}=A / B$ is completely reduced by $\Delta(\bar{A}, \bar{A})$, then $\operatorname{rad}(\bar{A})$ $=\operatorname{rad}(\bar{N})$ where $\bar{N}$ is the $J$-nucleus of $\bar{A}$. To show this, write the Levi decomposition $\bar{N}=\operatorname{rad}(\bar{N}) \oplus \bar{M}$ and now write $\bar{A}=\bar{N} \oplus J(\bar{A})$ $=\operatorname{rad}(\bar{N}) \oplus \bar{M} \oplus J(\bar{A})$. Now $A / \operatorname{rad}(\bar{N}) \cong \bar{M} \oplus J(\bar{A})$ is a semi-simple Malcev algebra, so by definition $\operatorname{rad}(\bar{A}) \subset \operatorname{rad}(\bar{N})$. But since $\bar{N}$ is an ideal of $A$, $\operatorname{rad}(\bar{N}) \subset \operatorname{rad}(\bar{A})$. Thus $\operatorname{rad}(\bar{N})=\operatorname{rad}(\bar{A})$. Secondly note that if $B$ is an ideal of $A$ and $B \subset \mathfrak{N}=\operatorname{rad}(A)$, then $\operatorname{rad}(A / B) \subset \mathfrak{N} / B$. For $A / \mathfrak{N} \cong(A / B) /(\mathfrak{N} / B)$ is semi-simple therefore by definition $\operatorname{rad}(A / B) \subset \mathfrak{N} / B$. Finally to show (1) we have by the preceding two remarks that $\operatorname{rad}(\bar{N})=\operatorname{rad}(\bar{A}) \subset \mathfrak{N} / \mathfrak{M}$. Now $\operatorname{rad}(\bar{N})$ is an ideal of $\bar{A}$, since $\operatorname{rad}(\bar{N})$ is an ideal of $\bar{N}$ and $\bar{N} J(\bar{A})=\overline{0}$. So $\operatorname{rad}(\bar{N})=W / \mathfrak{M}=\operatorname{rad}(\bar{A})$, where $W$ is an ideal of $A$ containing $\mathfrak{M}$. Therefore $A / W=(A / \mathfrak{M}) /(W / \mathfrak{M})=\bar{A} / \operatorname{rad}(\bar{A})$ is semi-simple and hence $\mathfrak{N} \subset W$. Thus $\mathfrak{R} / \mathfrak{M} \subset W / \mathfrak{M}=\operatorname{rad}(\bar{N})$; this proves (1). (2) Both follow from $\mathfrak{N} / \mathfrak{M}=\operatorname{rad}(\bar{N})$ $\subset \bar{N}$ which is a Lie algebra. (3) As in the proof of (1) write $A / \mathfrak{M}$ $=\operatorname{rad}(\bar{N}) \oplus \bar{M} \oplus J(\bar{A})=\mathfrak{N} / \mathfrak{M} \oplus \bar{M} \oplus J(\bar{A})$. (4) Let $\quad \bar{A}=A / \mathfrak{M}=\bar{N} \oplus J(\bar{A})$, then $\mathfrak{L}(\bar{A})=\mathfrak{L}(\bar{N} \oplus J(\bar{A}))=\mathfrak{L}(\bar{N}) \oplus \mathfrak{L}(J(\bar{A}))=R(\bar{N}) \oplus \mathfrak{L}(J(\bar{A}))$ $=R(\operatorname{rad}(\bar{N})) \oplus R(\bar{M}) \oplus \mathscr{L}(J(\bar{A}))$, by the Levi decomposition of $\bar{N}$. Thus $\mathcal{L}(\bar{A})=R(\operatorname{rad}(\bar{N})) \oplus \mathcal{L}(\bar{M}) \oplus \mathcal{L}(J(\bar{A}))=R(\operatorname{rad}(\bar{N})) \oplus \mathscr{L}(\bar{M} \oplus J(\bar{A}))$ $\cong R(\operatorname{rad}(\bar{N})) \oplus \mathscr{L}(A / \mathfrak{N})$. This last statement follows from $A / \mathfrak{M}$ $=\operatorname{rad}(\bar{N}) \oplus \bar{M} \oplus J(\bar{A})$ where $\operatorname{rad}(\bar{N})=\operatorname{rad}(\bar{A})=\mathfrak{N} / \mathfrak{M}$ and therefore $A / \mathfrak{N}$ $\cong(A / \mathfrak{M}) /(\mathfrak{N} / \mathfrak{M}) \cong \bar{M} \oplus J(\bar{A})$. Now $\mathscr{L}(A / \mathfrak{N})$ is semi-simple so we have $\operatorname{rad}(\mathscr{L}(\bar{A})) \subset R(\operatorname{rad}(\bar{N}))=\mathscr{L}(\operatorname{rad}(\bar{N}))=\mathfrak{L}(\mathfrak{N} / \mathfrak{M})$. For the reverse inclusion note that $\operatorname{rad}(\bar{N})$ is a solvable ideal of $\bar{A}$ and therefore $R(\operatorname{rad}(\bar{N}))$ is a solvable ideal of $\mathscr{L}(\bar{A})$. Hence $\mathscr{L}(\mathfrak{R} / \mathfrak{M})=R(\operatorname{rad}(\bar{N})) \subset \operatorname{rad}(\mathscr{L}(\bar{A}))$, since the radical of a Lie algebra is its maximal solvable ideal. Thus we have shown $\operatorname{rad}(\mathscr{L}(\bar{A}))$ $=R(\operatorname{rad}(\bar{N}))=\mathscr{L}(\overline{\mathfrak{N}})$ where $\bar{A}=A / \mathfrak{M}, \overline{\mathfrak{N}}=\mathfrak{N} / \mathfrak{M}$ and $\bar{N}$ is the $J$-nucleus of $\bar{A}$. (5) $(\mathcal{S}, \Delta(A, A)) \subset \operatorname{rad}(\mathscr{L}(A)) \cap \Delta(A, A)=\operatorname{rad}(\Delta(A, A))=\sigma$.

We shall now investigate invariant forms defined on a Malcev algebra and their relationships to the radical. Let $A$ be any nonassociative algebra over a field $F$. A function $f$ from $A \times A$ into $F$ is an invariant form for $A$ if (1) $f$ is bilinear; (2) $f(x, y)=f(y, x)$; (3) $f(x y, z)=f(x, y z)$. An invariant form $f$ is nondegenerate if $f(x, a)=0$ for all $a$ in $A$ implies $x=0$.

Theorem 7.16. Let $A$ be a Malcev algebra over a field $F$. The trace form $\operatorname{tr}(x, y)=\operatorname{trace}\left(R_{x} R_{y}\right)$ is an invariant form for $A$.

Proof. Clearly (1) and (2) are satisfied. (3) follows from (2.17).

The following lemmas are simple analogues of well-known properties of Lie algebras and their proofs will be omitted.

Lemma 7.17. Let $B$ be an ideal of a Malcev algebra $A$. The restriction of the 
trace form of $A$ to $B$ is identical with the trace form of $B$ considered as a Malcev algebra.

Lemma 7.18. Let $B$ be an ideal of a Malcev algebra $A$ such that $B^{2}=0$. Then $\operatorname{tr}(x, a)=0$ for every $x$ in $B, a$ in $A$.

We also have available Dieudonné's Theorem for arbitrary nonassociative algebras.

Theorem 7.19 (Dieudonné). Let $A$ be a finite dimensional algebra with nondegenerate invariant form $f$. Assume $A$ has no nonzero ideal $B$ with $B^{2}=0$. Then $A$ is a direct sum of simple ideals, an each of which $f$ is again nondegenerate.

Using Lemmas 7.17, 7.18 and Theorem 7.19 we have

TheOREM 7.20. Let $A$ be a Malcev algebra with a nondegenerate trace form, then $A$ is semi-simple.

Now let $\mathfrak{N}_{\mathrm{tr}}(A)=\{x \in A$ : for all $a$ in $A, \operatorname{tr}(x, a)=0\}$. Then $\mathfrak{N}_{\mathrm{tr}}(A)$ is an ideal of the Malcev algebra $A$ and we have

Corollary 7.21. If $B$ is an ideal of a Malcev algebra $A$ such that $\mathfrak{R}_{\mathrm{tr}}(A / B)$ $=\overline{0}$, then $\operatorname{rad}(A) \subset B$.

Proof. Since $\mathfrak{R}_{\mathrm{tr}}(A / B)=\overline{0}, f(\bar{a}, \bar{b})=\operatorname{tr}\left(R_{\bar{a}} R_{\bar{b}}\right)$ is a nondegenerate invariant form on $A / B$. Therefore $A / B$ is semi-simple and consequently $\operatorname{rad}(A) \subset B$. Note that $\operatorname{rad}(A)$ is contained in the minimal ideal $B$ of $A$ having the property stated in Corollary 7.21.

Combining some of the results of $\$ \$ 6$ and 7 we have

TheOREM 7.22. Let $A$ be a Malcev algebra of characteristic zero which is properly homomorphic to a semi-simple algebra and let $S$ be the maximal solvable ideal of $A$. Then (1) $S \subset \Im(A) \cap A$ s if $S$ is not a Lie algebra. (2) $J(S) \subset J(\Im(A)$ ) $\subset J(\Im(\Delta, A)) \subset A \sigma \subset A S \subset B$ where $B$ is the minimal such ideal of Corollary 7.21. (3) If $\Im(\Delta, A) \nsubseteq A \sigma$, then $\Im(\Delta, A) / A \sigma \subset \bar{N}$ where $\bar{N}$ is the J-nucleus of $\bar{A}=A / A \sigma$.

Proof. (1) follows from Theorem 6.5(4) and the fact that for an arbitrary algebra $A, S \subset \operatorname{rad}(A)$. (2) follows from Theorem 6.5(4), Proposition 6.9, Proposition 6.8 and Corollary 7.21. (3) follows from a modification of the proof of Theorem 6.6.

The preceding sections give fundamental results on the radical of a Malcev algebra and semi-simple Malcev algebras. However to complete a basic structure theory the simple non-Lie Malcev algebras must be determined. Further results by the author indicate that if $A$ is a finite dimensional simple non-Lie Malcev algebra over an algebraically closed field of characteristic zero, then $A$ is isomorphic to the algebra $A^{*}$ discussed in Example 3.2. 
8. Derivations of a Malcev algebra. A derivation of an algebra $A$ is a linear transformation $D$ of $A$ satisfying

$$
(x y) D=x D \cdot y+x \cdot y D
$$

for all $x, y$ in $A$. We may write (8.1) in an equivalent form

$$
R_{x D}=\left(R_{x}, D\right)
$$

for all $x$ in $A$.

Proposition 8.3. Let $A$ be a Malcev algebra. Then for every $x, y$ in $A$, the linear transformation $D(x, y)=\left(R_{x}, R_{y}\right)+R_{x y}$ is a derivation of $A$.

Proof. Beginning with (2.13), we have for any $x, y, z$ in $A, R_{x y \cdot s}=R_{y} R_{x x}$ $-R_{y z} R_{x}+R_{x} R_{y} R_{z}-R_{z} R_{x} R_{y}, R_{z y \cdot x}=R_{y} R_{x z}-R_{y x} R_{z}+R_{z} R_{y} R_{x}-R_{x} R_{z} R_{y}$ and $R_{x z} \cdot y$ $=R_{z} R_{y x}-R_{z y} R_{x}+R_{x} R_{z} R_{y}-R_{y} R_{z} R_{x}$. Adding these we obtain $R_{z D(y, x)}$ $=\left(R_{z}, D(y, x)\right)$. Thus $D(x, y)=-D(y, x)$ is a derivation of $A$ because of (8.2).

A set of elements $S$ of an algebra $A$ is characteristic if $S D \subset S$ for all derivations $D$ of $A$.

Proposition 8.4. Let $A$ be a Malcev algebra. Then $J(A)$ and $N$ are characteristic ideals of $A$.

Proof. A simple calculation shows that $J(x, y, z) D=J(x D, y, z)+J(x, y D, z)$ $+J(x, y, z D)$. Now the results follow from Theorem 3.5 and the definition of $J$-nucleus.

TheOREM 8.5. Let $A$ be a Malcev algebra, then $R_{x}$ is a derivation of $A$ if and only if $x$ is in $N$.

Proof. $R_{x}$ is a derivation if and only if $a b \cdot x=(a b) R_{x}=a R_{x} \cdot b+a \cdot b R_{x}$ $=a x \cdot b+a \cdot b x$, that is, $0=J(a, b, x)$ for all $a, b$ in $A$; that is, if and only if $x$ is in $N$.

Corollary 8.6. Let $A$ be a simple Malcev algebra, then $R_{x} \neq 0$ is a derivation of $A$ if and only if $A=N$.

The derivations of a Malcev algebra $A$ form a Lie algebra $D$ under commutation which we shall now investigate. Let $D$ be any derivation of a Malcev algebra $A$ and rewrite (8.2) as $R_{x} D=R_{x D}+D R_{x}$, for any $x$ in $A$. Then $R_{x} R_{y} D$ $=R_{x}\left(R_{y D}+D R_{y}\right)=R_{x} R_{y D}+R_{x D}+D R_{x} R_{y}$, or $\left(R_{x} R_{y}, D\right)=R_{x} R_{y D}+R_{x D} R_{y}$. Similarly $-\left(R_{y} R_{x}, D\right)=-R_{y D} R_{x}-R_{y} R_{x D}$. Since $\left(R_{x y}, D\right)=R_{(x y) D}=R_{x \cdot y D}+R_{x D \cdot y}$, we may add these last three equations to obtain

$$
(D(x, y), D)=D(x D, y)+D(x, y D)
$$

where $D(u, v)$ is the derivation given in Theorem 8.3. Formula (8.7) can be iterated to obtain

$$
\left(\cdots \left((D(x, y), \overbrace{D), D), \cdots), D}^{n})=\sum_{k=0}^{n} C_{n, k} D\left(x D^{n-k}, y D^{k}\right) .\right.\right.
$$


As a consequence of (8.7) we have

TheOREM 8.9. Let $D$ be the derivation algebra of a Malcev algebra $A$ and let $g$ be the subspace of $D$ generated by all derivations of $A$ of the form $D(x, y)$. Then $\mathrm{g}$ is an ideal of $D$.

Schafer [8] has defined a derivation $D$ of an arbitrary nonassociative algebra $A$ to be inner if $D \in \mathscr{L}(A)$. We have

Theorem 8.10. Let $A$ be a Malcev algebra. Then a derivation $D$ of $A$ is inner if and only if $D=R_{n}+\sum_{i} D\left(x_{i}, y_{i}\right)$ for some $n \in N$ and $x_{i}, y_{i} \in A$.

Proof. Let $D=R_{z}+\sum_{i}\left(R_{x_{i}}, R_{y_{i}}\right)$ be a derivation contained in $\mathscr{L}(A)$. We know that $D_{1}=\sum_{i} R_{x_{i} y_{i}}+\left(R_{x_{i}}, R_{y_{i}}\right)$ is a derivation of $A$. Hence $D-D_{1}=R_{z}$ must be a derivation of $A$. From Theorem 8.5 we obtain $z-\sum_{i} x_{i} y_{i}=n \in N$.

Corollary 8.11. Let $A$ be a Malcev algebra such that for every $n \in N$, $R_{n}=0$. Then $D$ is an inner derivation if and only if $D=\sum_{i} D\left(x_{i}, y_{i}\right)$.

We shall now consider relationships between $\Delta(A, A)$ and derivations. The linear transformations $\Delta(x, y)=\left(R_{x} R_{y}\right)-R_{x y}$ and the derivations $D(x, y)$ $=\left(R_{x}, R_{y}\right)+R_{x y}$ yield equations

$$
\begin{aligned}
& D(x, y)+\Delta(x, y)=2\left(R_{x}, R_{y}\right), \\
& D(x, y)-\Delta(x, y)=2 R_{x y} .
\end{aligned}
$$

First we have

Proposition 8.14. Let $D$ be the derivation algebra of a Malcev algebra $A$. Then $(\Delta(A, A), D) \subset \Delta(A, A)$.

Proof. Let $\Delta(x, y)$ be in $\Delta(A, A)$ and $D$ be a derivation, then $(\Delta(x, y), D)$ $=\left(D(x, y)-2 R_{x y}, D\right)=(D(x, y), D)-2\left(R_{x y}, D\right)=D(x D, y)+D(x, y D)$ $-2 R_{(x y) D}=D(x D, y)-2 R_{x D \cdot y}+D(x, y D)-2 R_{x \cdot y D}=\Delta(x D, y)+\Delta(x, y D)$. By linearity we obtain $(\Delta(A, A), D) \subset \Delta(A, A)$.

TheORem 8.15. If $A$ is a simple, non-Lie, Malcev algebra, then $\Delta(A, A)$ $=g \oplus R(A)$, where this is a vector space direct sum.

Proof. This follows from (8.13), $A=A^{2}$ is simple and Theorem 8.5.

Theorem 8.16. Let $x_{i}, y_{i}(i=1, \cdots, n)$ be elements of a Malcev algebra $A$. Then $\Delta=\sum_{i=1}^{n} \Delta\left(x_{i}, y_{i}\right.$ is a derivation of $A$ if and only if $\sum_{i=1}^{n} x_{i} y_{i}$ is in $N$.

Proof. This follows from an extension of (8.13) and Theorem 8.5.

Corollary 8.17. Let $x, y$ be elements of a simple, non-Lie, Malcev algebra; then $\Delta(x, y)$ is a derivation of $A$ if and only if $x y=0$, that is, if and only if $\Delta(x, y)=D(x, y)$. 
CoRollary 8.18. Let $x, y$ be elements of a simple Malcev algebra $A$ with $R_{x y} \neq 0$; then $\Delta(x, y)$ is a derivation of $A$ if and only if $A=N$.

Corollary 8.19. Let $A$ be a Malcev algebra such that for every $x, y$ in $A$, $\Delta(x, y)$ is a derivation. Then $J^{2}(A)=0$.

We shall conclude this section with a discussion of derivations of the $A^{*}$ algebra given in Example 3.2. A somewhat lengthy but straightforward calculation reveals that every derivation of $A^{*}$ has the matrix form (relative to the $e_{i}$ basis),

$$
D=\left[\begin{array}{rcccccc}
0 & 2 d_{2} & 2 d_{3} & 2 d_{4} & -2 d_{5} & -2 d_{6} & -2 d_{7} \\
d_{5} & d_{8} & d_{9} & d_{10} & 0 & d_{4} & -d_{3} \\
d_{6} & d_{11} & d_{12} & d_{13} & -d_{4} & 0 & d_{2} \\
d_{7} & d_{14} & d_{15} & -d_{8}-d_{12} & d_{3} & -d_{2} & 0 \\
-d_{2} & 0 & -d_{7} & d_{6} & -d_{8} & -d_{11} & -d_{14} \\
-d_{3} & d_{7} & 0 & -d_{5} & -d_{9} & -d_{12} & -d_{16} \\
-d_{4} & -d_{6} & d_{5} & 0 & -d_{10} & -d_{13} & d_{8}+d_{12}
\end{array}\right]
$$

From the definitions, every derivation of an algebra $A$ is a derivation of $A^{(-)}$. It follows from the work of Schafer [8] that the derivation Lie algebra of $A^{*}$ is the exceptional Lie algebra $G_{2}$. This might certainly be suspected from the 14 parameter form of $D$ in (8.20). Now using Theorem 8.9 and the fact that $G_{2}$ is a simple Lie algebra, every derivation of $A^{*}$ is inner and every $D(x, y)$ has the form (8.20). A somewhat lengthy calculation yields that $\Delta\left(A^{*}, A^{*}\right)$ is a simple Lie algebra of dimension 21 .

If we compute $R_{x}$ for an element $x=\sum_{i=1}^{7} x_{i} e_{i}$ of $A^{*}$, we have

$$
R_{x}=\left[\begin{array}{rrrrrrr}
0 & 2 x_{2} & 2 x_{3} & 2 x_{4} & -2 x_{5} & -2 x_{6} & -2 x_{7} \\
x_{5} & -2 x_{1} & 0 & 0 & 0 & -2 x_{4} & 2 x_{3} \\
x_{6} & 0 & -2 x_{1} & 0 & 2 x_{4} & 0 & -2 x_{2} \\
x_{7} & 0 & 0 & -2 x_{1} & -2 x_{3} & 2 x_{2} & 0 \\
-x_{2} & 0 & 2 x_{7} & -2 x_{6} & 2 x_{1} & 0 & 0 \\
-x_{3} & -2 x_{7} & 0 & 2 x_{5} & 0 & 2 x_{1} & 0 \\
-x_{4} & 2 x_{6} & -2 x_{5} & 0 & 0 & 0 & 2 x_{1}
\end{array}\right]
$$

If we let $y=\sum_{i=1}^{7} y_{i} e_{i}$, then we may consider $R_{y}$ as (8.21) with $x_{i}$ replaced by $y_{i}$. A straightforward computation reveals that $D(x, y)$ has the form (8.20) as noted above. If we let $R(i, j)=x_{i} y_{j}-x_{j} y_{i}$, then the parameters for $D(x, y)$ in $(8.20)$ are 


$$
\begin{array}{ll}
d_{2}=-4 R(2,1)-2 R(7,6), & d_{9}=6 R(5,3), \\
d_{8}=-4 R(3,1)-2 R(5,7), & d_{10}=6 R(5,4), \\
d_{4}=-4 R(4,1)-2 R(6,5), & d_{11}=6 R(6,2), \\
d_{6}=4 R(5,1)+2 R(4,3), & d_{12}=2 R(4,7)+2 R(2,5)+4 R(6,3), \\
d_{6}=4 R(6,1)+2 R(2,4), & d_{18}=6 R(6,4), \\
d_{7}=4 R(7,1)+2 R(3,2), & d_{14}=6 R(7,2), \\
d_{8}=2 R(4,7)+2 R(3,6)+4 R(5,2), & d_{16}=6 R(7,3) .
\end{array}
$$

As a specific example we take $x=e_{2}+2 e_{3}+e_{4}+e_{5}-e_{6}$ and $y=e_{3}+e_{4}$ and find

$$
D(x, y)=\left[\begin{array}{rrrrrrr}
0 & 0 & 0 & 0 & 4 & -4 & 4 \\
-2 & 2 & 6 & 6 & 0 & 0 & 0 \\
2 & 0 & -4 & -6 & 0 & 0 & 0 \\
-2 & 0 & 0 & 2 & 0 & 0 & 0 \\
0 & 0 & 2 & 2 & -2 & 0 & 0 \\
0 & -2 & 0 & 2 & -6 & 4 & 0 \\
0 & -2 & -2 & 0 & -6 & 6 & -2
\end{array}\right]
$$

The root spaces for the derivation (8.22) are

$$
\begin{aligned}
L_{0} & =\left\{e_{1}+2 e_{5}-2 e_{6}+2 e_{7}\right\}, & & L_{2}=\left\{e_{2}+e_{3}, e_{1}-e_{4}+e_{5}-e_{6}+e_{7}\right\}, \\
L_{-2} & =\left\{e_{5}-e_{6}+e_{7}, e_{3}+e_{4}+e_{6}\right\}, & & L_{4}=\left\{e_{2}+e_{3}+e_{5}+e_{6}\right\}, \\
L_{-4} & =\left\{e_{3}+e_{4}\right\} . & &
\end{aligned}
$$

We note that in this example the root spaces are not one-dimensional. Thus, it appears that the decomposition of a Malcev algebra relative to a derivation does yot yield a theory analogous to that of Lie algebras. A complete investigation of this possibility has not been carried out.

Finally we note that the invariant form $f(x, y)=\operatorname{trace}\left(R_{x} R_{y}\right)$ is given by $f(x, y)=6\left(4 x_{1} y_{1}+2 x_{2} y_{6}+2 x_{3} y_{6}+2 x_{4} y_{7}+2 x_{6} y_{2}+2 x_{6} y_{3}+2 x_{7} y_{4}\right)$. We see that $f(x, y)$ is nondegenerate on $A^{*}$ for characteristic not 2 or 3 .

9. The holomorph of a Malcev algebra. The holomorph of a Lie algebra $A$ is the linear space $\mathfrak{H C}=A \oplus \mathbb{D}=\{a+D: a \in A$ and $D \in \mathscr{D}\}$ where $D$ is the derivation algebra of $A$ and multiplication * is defined by

$$
\left(a+D_{1}\right) *\left(b+D_{2}\right)=a b+a D_{2}-b D_{1}+\left(D_{1}, D_{2}\right) .
$$

This now makes $\mathfrak{T}$ into a Lie algebra.

We use similar definitions to form the holomorph of a Malcev algebra $A$. However, the following theorem indicates that the concept has limitations in the study of Malcev algebras. 
THEOREM 9.1. The holomorph of a Malcev algebra is a Malcev algebra if and only if for all $x, y, z$ in $A$ and $D$ in $D, J(x, y, z) D=J(x, y, D z)$ where we define $D z=-z D$.

Proof. It is clear that the holomorph of the Malcev algebra $A$ will be a Malcev algebra if and only if the identity

$$
J(x, y, w z)+J(w, y, x z)=J(x, y, z) w+J(w, y, z) x
$$

is satisfied when any or all of the parameters $x, y, z, w$ are replaced by derivations of the algebra.

If $R$ and $S$ are derivations, we note that $J(R, S, z)=J(R, y, S)=J(x, R, S)$ $=0$. Also $J(R, y, z)=J(x, R, z)=J(x, y, R)=0$. Thus it is clear that if 2,3 , or 4 of the parameters $x, y, z, w$ in (2.7) are derivations, the identity is satisfied.

Now we consider the case when only one parameter is a derivation. If $y=R$, the identity (2.7) is again satisfied. If $x=R$, we have

$$
J(w, y, R z)=J(w, y, z) R,
$$

and a similar identity holds for $w=R$. If $z=R$, we have the identity

$$
J(x, y, w R)+J(w, y, x R)=0 .
$$

Since (9.2) implies (9.3), the proof is complete.

In a manner similar to that for the holomorph, the linear space $A \oplus \Delta(A, A)$ can be made into an algebra by defining $\left(A+\Delta_{1}\right) *\left(b+\Delta_{2}\right)=a b+a \Delta_{2}-b \Delta_{1}$ $+\left(\Delta_{1}, \Delta_{2}\right)$ where $a, b$ in $A, \Delta_{1}, \Delta_{2}$ in $\Delta(A, A)$. Now note that if $D$ is replaced by $\mathcal{G}$ in Theorem 9.1 we obtain $A \oplus \mathscr{S}$ is a Malcev algebra if and only if

$$
J(x, y, z) D=J(x, y, D z)
$$

for every $D$ in $g$ and every $x, y, z$ in $A$. Also note that if $A=J(A)$, then $\checkmark \subset \Delta(A, A)$. We use these facts in the following

TheOREM 9.5. If $A$ is a Malcev algebra such that $A=J(A)$ and if $A \oplus \Delta(A, A)$ is a Malcev algebra, then $\Delta(x, y)^{2}=0$ for every $x, y$ in $A$.

Proof. Suppose $A \oplus \Delta(A, A)$ is a Malcev algebra, then by (8.13), (9.4) and the preceding remarks, we have for all $x, y, z$ in $A$

$$
J(x, y, z)\left(\Delta(x, y)+2 R_{x y}\right)=-J\left(x, y, z\left(\Delta(x, y)+2 R_{x y}\right)\right)
$$

that is, $\Delta(x, y)^{2}+2 \Delta(x y) R_{x y}=-\Delta(x, y)^{2}-2 R_{x y} \Delta(x, y)$. Thus we have $\Delta(x, y)^{2}$ $=-\Delta(x, y) R_{x y}-R_{x y} \Delta(x, y)$. But from (2.33) we have $\Delta(x, y)^{2}=-3 \Delta(x, y) R_{x y}$ and therefore $2 \Delta(x, y) R_{x y}=R_{x y} \Delta(x, y)$. This identity together with (2.26) yield $2 J(z, x, y) \cdot x y=J(z \cdot x y, x, y)=z J(x y, x, y)+J(z, x, y) \cdot x y-2 J(x y, z, x y)$, and therefore $J(z, x, y) \cdot x y=z J(x y, x, y)=0$. Thus $0=J(z, x, y) \cdot x y=z \Delta(x, y) R_{x y}$ and therefore $\Delta(x ; y)^{2}=0$. 


\section{Bibliography}

1. A. A. Albert, The radical of a non-associative algebra, Bull. Amer. Math. Soc. vol. 48 (1942) pp. 891-898.

2. R. H. Bruck and E. Kleinfeld, The structure of alternatioe division rings, Proc. Amer. Math. Soc. vol. 2 (1951) pp. 878-890.

3. N. Jacobson, Structure of rings, Amer. Math. Soc. Colloquium Publications, vol. 37, 1956, pp. 200-202.

4. - Completely reducible Lie algebras of linear transformations, Proc. Amer. Math. Soc. vol. 2 (1951) pp. 105-113.

5. - Lectures in abstract algebras, vol II, New York, D. Van Nostrand Co., 1953.

6. E. Kleinfeld, $A$ note on Moufang-Lie rings, Proc. Amer. Math. Soc. vol. 9 (1958) pp. 72-74.

7. A. Malcev, Analytic loops, Mat. Sb. vol. 78 (1955) pp. 569-578.

8. R. Schafer, Inner derivations of non-associatioe algebras, Bull. Amer. Math. Soc. vol. 55 (1949) pp. 769-776.

University of Chichgo,

Chicago, Illinors 